Discrete Comput Geom 29:175-209 (2003)

DOI: $10.1007 / \mathrm{s} 00454-002-2820-6$

\title{
Substitution Delone Sets
}

\author{
Jeffrey C. Lagarias ${ }^{1}$ and Yang Wang ${ }^{2}$ \\ ${ }^{1}$ AT\&T Labs - Research, \\ Florham Park, NJ 07932-0971, USA \\ jcl@ research.att.com \\ ${ }^{2}$ School of Mathematics, Georgia Institute of Technology, \\ Atlanta, GA 30332, USA \\ wang@math.gatech.edu
}

Abstract. Substitution Delone set families are families of Delone sets $\mathcal{X}=\left(X_{1}, \ldots, X_{n}\right)$ which satisfy the inflation functional equation

$$
X_{i}=\bigvee_{j=1}^{m}\left(\mathrm{~A}\left(X_{j}\right)+\mathcal{D}_{i j}\right), \quad 1 \leq i \leq m,
$$

in which $A$ is an expanding matrix, i.e., all of the eigenvalues of $A$ fall outside the unit circle. Here the $\mathcal{D}_{i j}$ are finite sets of vectors in $\mathbb{R}^{d}$ and $\bigvee$ denotes union that counts multiplicity.

This paper characterizes families $\mathcal{X}=\left(X_{1}, \ldots, X_{n}\right)$ that satisfy an inflation functional equation, in which each $X_{i}$ is a multiset (set with multiplicity) whose underlying set is discrete. It then studies the subclass of Delone set solutions, and gives necessary conditions on the coefficients of the inflation functional equation for such solutions $\mathcal{X}$ to exist. It relates Delone set solutions to a narrower subclass of solutions, called self-replicating multi-tiling sets, which arise as tiling sets for self-replicating multi-tilings.

\section{Introduction}

Aperiodic and self-similar structures in $\mathbb{R}^{d}$ have been extensively studied using tilings of $\mathbb{R}^{d}$ as models. Among these are the classes of self-similar tilings and, more generally, selfaffine tilings. Such tilings have been proposed as models for quasicrystalline structures [2], [6], [7], [22], [24], [32]; they also arise in constructions of compactly supported wavelets and multiwavelets [1], [4], [5]. An alternate method of modeling quasicrystalline structures uses discrete sets, more specifically Delone sets (defined below), see [14]-[16], [26]-[28], and [33]. These sets model the atomic positions occupied in the structure. In terms of tilings, such discrete sets can be viewed either as tiling sets, representing the 
translations used in forming tilings by translation of a finite number of different prototile types, or as control points marking in some way the location of each tile.

Comparison of these two types of models, which appear rather different, motivates the question: Is there a notion of self-similarity appropriate to discrete sets and Delone sets? This paper develops such a notion, which is based on a system of functional equations dual to the functional equations associated to self-affine tilings and multi-tilings.

We first recall the functional equation associated to the construction of finite sets of tiles $\left\{T_{1}, \ldots, T_{n}\right\}$ which tile $\mathbb{R}^{d}$ with special kinds of self-affine tilings. The tiles are solutions to a finite system of set-valued functional equations which we call multi-tile equations, which encode a self-affine property. An inflation map $\varphi: \mathbb{R}^{d} \rightarrow \mathbb{R}^{d}$ is an expanding linear map $\varphi(\mathbf{x})=\mathbf{A} \mathbf{x}$ in which $\mathrm{A}$ is an expanding $n \times n$ real matrix, i.e., all its eigenvalues $|\lambda|>1$. Let $\left\{\mathcal{D}_{i j}: 1 \leq i, j \leq n\right\}$ be finite sets in $\mathbb{R}^{d}$ called digit sets.

Multi-Tile Functional Equation. The family of compact sets $\left(T_{1}, T_{2}, \ldots, T_{n}\right)$ satisfies the system of equations

$$
\mathrm{A}\left(T_{i}\right)=\bigcup_{j=1}^{n}\left(T_{j}+\mathcal{D}_{j i}\right)
$$

for $1 \leq i \leq n$

This functional equation is set-valued, i.e., points are counted without multiplicity in the set union on the right side of (1.1). The subdivision matrix associated to (1.1) is

$$
\mathrm{S}=\left[\left|\mathcal{D}_{i j}\right|\right]_{1 \leq i, j \leq n} .
$$

These functional equations have a nice solution theory when the substitution matrix satisfies the following extra condition.

Definition 1.1. A nonnegative real matrix $S$ is primitive if some power $S^{k}$ has strictly positive entries.

In the one-dimensional case the subdivision matrix associated to (1.1) is always primitive. It is known that when $S$ is primitive the functional equation (1.1) has a unique nonempty solution

$$
\mathcal{T}:=\left(T_{1}, \ldots, T_{n}\right)
$$

in which all $T_{i}$ are compact sets (see Theorem 2.3 of [1] and see [4]); in this case all $T_{i}$ are nonempty. In the imprimitive case there can be more than one nonempty solution $\mathcal{T}:=\left(T_{1}, \ldots, T_{n}\right)$ in which all $T_{i}$ are compact sets (see examples in [4]), and the theory becomes more complicated. There are however at most finitely many such solutions, and under general conditions there is a unique "maximal" compact solution, in which all $T_{i}$ are nonempty, see [1]. In this paper we primarily consider functional equations where the subdivison matrix $S$ is primitive, though some results apply for general $S$.

We are interested in the case where these sets $T_{i}$ have positive Lebesgue measure and can be used in tiling $\mathbb{R}^{n}$ by a self-affine tiling. The tiling sets for such tilings are special solutions to a second functional equation involving the same data, which is "adjoint" to 
the multi-tile functional equation. This functional equation counts multiplicities of sets, unlike (1.1), and we consider solutions to it that are multisets.

Inflation Functional Equation. The multiset family $\mathcal{X}:=\left(X_{1}, X_{2}, \ldots, X_{n}\right)$ satisfies the system of equations

$$
X_{i}=\bigvee_{j=1}^{n}\left(\mathrm{~A}\left(X_{j}\right)+\mathcal{D}_{i j}\right), \quad 1 \leq i \leq n,
$$

where $\mathcal{D}_{i j}$ are finite sets of vectors in $\mathbb{R}^{d}$.

Here each $X_{i}$ is a multiset and $\bigvee_{j=1}^{n}$ denotes multiset union, as defined in Section 2. We can view the inflation functional equation as a fixed point equation $\psi(\mathcal{X})=\mathcal{X}$, where $\psi(\cdot)$ is an operator taking multisets to multisets, defined by $\psi(\mathcal{X}):=\left(X_{1}^{\prime}, \ldots, X_{n}^{\prime}\right)$ with

$$
X_{i}^{\prime}:=\bigvee_{j=1}^{n}\left(\mathrm{~A}\left(X_{j}\right)+\mathcal{D}_{i j}\right)
$$

The inflation functional equation can also be written as a system of equations for the multiplicity functions:

$$
\begin{aligned}
m_{X_{i}}(\mathbf{x})= & \sum_{j=1}^{n} m_{\mathrm{A}\left(X_{j}\right)+\mathcal{D}_{i j}}(\mathbf{x})=\sum_{j=1}^{n} \sum_{\mathbf{d} \in \mathcal{D}_{i j}} m_{X_{j}}\left(\mathrm{~A}^{-1}(\mathbf{x}-\mathbf{d})\right), \\
& \text { for all } \quad \mathbf{x} \in \mathbb{R}^{d} .
\end{aligned}
$$

A self-replicating multi-tiling consists of a pair $(\mathcal{T}, \mathcal{X})$ of solutions to the multi-tile functional equation and the inflation functional equation such that:

(1) The solution $\mathcal{T}:=\left(T_{1}, \ldots, T_{n}\right)$ to the multi-tile functional equation has all sets $T_{i}$ of positive Lebesgue measure.

(2) The solution $\mathcal{X}=\left(X_{1}, \ldots, X_{n}\right)$ to the inflation functional equation are sets (multisets with all multiplicities one) and $\bigcup_{i=1}^{n}\left(T_{i}+X_{i}\right)$ is a tiling of $\mathbb{R}^{d}$, using the $T_{i}$ 's as prototiles.

This notion extends the notion of self-replicating tilings studied by Kenyon [8], [9], which allow only one type of tile $(n=1)$. Later studies [10], [11] allowed $n$ tile types but restricted the inflation matrix $A$ to be a similarity.

We call a family $\mathcal{X}$ a self-replicating multi-tiling family (for a fixed inflation functional equation) if it appears as part of a self-replicating multi-tiling $(\mathcal{T}, \mathcal{X})$. It follows that each multiset $X_{i}$ in such a family is a uniformly discrete set. There are very strong restrictions on the data $\left(\mathrm{A}, \mathcal{D}_{i j}\right)$ on the functional equations for a self-replicating multi-tiling to exist, for example, the Perron eigenvalue condition given below.

This paper studies solutions to the inflation functional equation that are discrete, including solutions that do not correspond to tilings. The inflation functional equation has properties that significantly differ from those of the multi-tile functional equation. 
For example, the multi-tile functional equation has a unique solution (in the case of primitive subdivision matrix) because the solution $\mathcal{T}$ is given by the unique fixed point of a contracting system of mappings. In contrast, the inflation functional equation involves an expanding system of mappings, and its solutions are not compact sets. It may have infinitely many different solutions, even infinitely many very nice solutions in some cases. Our replacement for the "contracting" condition is to restrict attention to solutions $\mathcal{X}=\left(X_{1}, \ldots, X_{n}\right)$ having the property that all $X_{j}$ are discrete multisets. A set $\underline{X}$ in $\mathbb{R}^{d}$ is discrete if each bounded set in $\mathbb{R}^{d}$ contains finitely many elements of $\underline{X}$. A multiset $X$ is discrete if its underlying set $\underline{X}$ is discrete and each element in $X$ has a finite multiplicity. A multiset family $\mathcal{X}$ is discrete if each multiset $X_{i}$ in it is discrete.

In Section 2 we give precise definitions and statements of the main results in the paper. Below we summarize the general contents of the other sections.

In Section 3 we develop a structure theory for multiset solutions to the inflation functional equation that are discrete. These results are proved for general inflation functional equations, with no primitivity restriction. We show that discrete multiset solutions decompose uniquely into a finite number of irreducible discrete multisets, and that each irreducible discrete multiset is characterized by a finite set of points in it. However, there are significant restrictions on the inflation functional equation data $\left(\mathrm{A}, \mathcal{D}_{i j}\right)$ necessary for the existence of any discrete multiset solution, as indicated in later sections.

In Sections 4-7 we study multiset solutions which correspond more closely to tilings. A multiset $X$ is weakly uniformly discrete if there is a positive radius $r$ and a finite constant $m \geq 1$ such that each ball of radius $r$ contains at most $m$ points of $X$, counting multiplicities; it is relatively dense if there is a radius $R$ such that each ball of radius $R$ contains at least one point of $X$. A multiset $X$ is a weak Delone set $X$ if it is weakly uniformly discrete and relatively dense.

A solution $\mathcal{X}=\left(X_{1}, \ldots, X_{n}\right)$ to the inflation functional equation is a weak substitution Delone multiset family if each $X_{i}$ is a weak Delone multiset. In studying solutions which are weak Delone multisets, we restrict attention to inflation functional equations that have a primitive subdivision matrix $\mathrm{S}$. Then we make use of Perron-Frobenius theory, which asserts that a primitive nonnegative real matrix $\mathrm{M}$ has a positive real eigenvalue $\lambda(\mathrm{M})$ such that:

(i) $\lambda(M)$ has multiplicity one.

(ii) $\lambda(M)>\left|\lambda^{\prime}\right|$ for all eigenvalues $\lambda^{\prime}$ of $M$ with $\lambda^{\prime} \neq \lambda(M)$.

(iii) $M$ has both right and left eigenvectors for eigenvalue $\lambda(M)$ which have positive real entries.

We call $\lambda(\mathrm{M})$ the Perron eigenvalue of $\mathrm{M}$; it is equal to the spectral radius of $\mathrm{M}$.

For inflation functional equations whose substitution matrix is primitive, we show that those multiset equations having a weak Delone set solution must satisfy the following:

Perron Eigenvalue Condition. The Perron eigenvalue $\lambda(\mathrm{S})$ of the subdivision matrix $\mathrm{S}$ satisfies

$$
\lambda(S)=|\operatorname{det}(A)| .
$$


More generally, there are inequalities relating the Perron eigenvalue and properties of solutions of the multi-tile and inflation functional equations, as follows:

(1) A necessary condition for the multi-tile functional equation with primitive subdivision matrix to have $\mathcal{T}=\left(T_{1}, \ldots, T_{n}\right)$ with some (and hence all) $T_{i}$ of positive Lebesgue measure is that

$$
\lambda(S) \geq|\operatorname{det}(A)|
$$

(2) A necessary condition for the inflation functional equation with primitive subdivision matrix to have a solution $\mathcal{X}=\left(X_{1}, \ldots, X_{n}\right)$ with some (and hence all) $X_{i}$ weakly uniformly discrete is that

$$
\lambda(\mathrm{S}) \leq|\operatorname{det}(\mathrm{A})|
$$

Inequality (1) is established by taking the Lebesgue measure on both sides of the multi-tile equation (1.1), see Theorem 5.2. In the special case when $A$ is a similarity Mauldin and Williams [25] showed the stronger result that if $\lambda(S)<|\operatorname{det}(A)|$, then the Hausdorff dimension of each $T_{i}$ must be less than $d$. Inequality (2) is established in Theorem 4.3.

In Section 5, under the assumption that the Perron eigenvalue condition holds, we give necessary and sufficient conditions for the associated multi-tiling equation to have solutions $T_{i}$ having positive Lebesgue measure. In Section 6 we use these to show that the existence of weak substitution Delone set solutions $\psi(\mathcal{X})=\mathcal{X}$ ) is closely associated with self-replicating multi-tilings. In particular, the associated multi-tile functional equation then necessarily has a compact solution $\mathcal{T}=\left(T_{1}, T_{2}, \ldots, T_{n}\right)$ with the $T_{i}$ all having positive Lebesgue measure, and some iterate $\psi^{N}(\cdot)$ of the inflation functional equation has a solution that is a self-replicating multi-tiling. In Section 7 we supplement this with a sufficient condition to have a self-replicating multi-tiling.

In Section 8 we give examples showing the limits of our results.

We distinguish multi-tilings, which are tilings of $\mathbb{R}^{d}$ using several types of tiles, from multiple tilings, which are arrangements of tiles in $\mathbb{R}^{d}$ such that almost all points in $\mathbb{R}^{d}$ are covered exactly $p$ times, for some positive integer $p$. We call $p$ the thickness of the multiple tiling, and sometimes call such a tiling $p$-thick. A 1-thick tiling is just an ordinary tiling. The constructions of this paper can have associated to them tilings which are multiple in both senses, that is, $p$-thick multi-tilings for some $p \geq 2$. By definition self-replicating multi-tilings are 1-thick tilings. We remark that the tiling set of a $p$-thick tiling cannot in general be partitioned to give a union of 1-thick tilings, even in the one-dimensional case.

To conclude this introduction, given any Delone set $X$ one can associate a topological dynamical system $\left([[X]], \mathbb{R}^{d}\right)$ with an $\mathbb{R}^{d}$-action, in which $[[X]]$ is the closure of the orbit of $X$ under the $\mathbb{R}^{d}$-translation action in an appropriate topology, see [37]. For substitution Delone set families these dynamical systems can be viewed as a generalization of substitution dynamical systems [31], in that every primitive substitution dynamical system is topologically conjugate to a suitable substitution Delone set dynamical system. Under sufficiently strong extra hypotheses substitution Delone set dynamical systems are minimal and uniquely ergodic. We hope to return to this question elsewhere. 
Notation. The positive integer $d$ refers to the dimension $\mathbb{R}^{d}$. We denote the closed Euclidean ball of radius $R$ around $\mathbf{x}$ by $B_{R}(\mathbf{x}):=\left\{\mathbf{y} \in \mathbb{R}^{d}:\|\mathbf{y}-\mathbf{x}\| \leq R\right\}$. The positive integer $n$ denotes the number of tile types or terms in the inflation functional equation. The positive integer $p$ refers to the period of a cycle, and also sometimes to the the thickness of a multiple covering or tiling of $\mathbb{R}^{d}$.

\section{Definitions and Main Results}

We consider solutions to the inflation functional equation that are multisets.

Definition 2.1. (i) A multiset $X$ in $\mathbb{R}^{d}$ is a set $\underline{X}$ together with a positive integer-valued function, the multiplicity function $m_{X}: \underline{X} \rightarrow \mathbb{Z}_{>0}$, which assigns to each element $\mathbf{x} \in \underline{X}$ a "multiplicity" $m_{X}(\mathbf{x})$. Given a multiset $X$ we use $\underline{X}$ to denote the underlying set of $X$, i.e., without counting the multiplicity, and use either $m_{X}(\mathbf{x})$ or $m(X, \mathbf{x})$ to denote the multiplicity function. A multiset is called an ordinary set if every element in $X$ has multiplicity one.

(ii) We extend the multiplicity function to all $\mathbf{x} \in \mathbb{R}^{d}$ by setting $m_{X}(\mathbf{x})=0$ if $\mathbf{x} \notin \underline{X}$.

(iii) Given multisets $X_{1}$ and $X_{2}$ in $\mathbb{R}^{d}$ we say $X_{1} \subseteq X_{2}$ provided $\underline{X}_{1} \subseteq \underline{X}_{2}$ and $m_{X_{1}}(\mathbf{x}) \leq m_{X_{2}}(\mathbf{x})$ for all $\mathbf{x} \in X_{2}$. In particular, $\underline{X} \subseteq X$.

The term "multiset" is attributed to N. G. deBruijn (see p. 636 of [13]) and the concept has found many uses. The combinatorial view of a multiset is as a collection of possibly repeated elements. For example, $X=\{0,0,1,3,4,4,4\}$ represents a multiset in $\mathbb{R}$ in which 0 is counted twice and 4 is counted three times. Thus $\underline{X}=\{0,1,3,4\}$ and

$$
m_{X}(0)=2, \quad m_{X}(1)=m_{X}(3)=1, \quad m_{X}(4)=3 .
$$

One may also regard a multiset $X$ in $\mathbb{R}^{d}$ as a nonnegative integer-valued pure discrete measure on $\mathbb{R}^{d}$. The results of this paper could be alternatively formulated in a measuretheoretic framework, and the inflation functional equation then expresses an equality of measures. A reviewer observed that a measure-theoretic treatment could prove useful for further generalizations, but we do not attempt it here.

Definition 2.2. For any multisets $X$ and $Y$ the multiset union $X \vee Y$ is the multiset having multiplicity function

$$
m_{X \vee Y}:=m_{X}+m_{Y}
$$

and the multiset intersection $X \wedge Y$ is the multiset having multiplicity function

$$
m_{X \wedge Y}:=\min \left\{m_{X}, m_{Y}\right\} .
$$

For a multiset $X$ and a set (or multiset) $\mathcal{D}$ the multiset sum $X+\mathcal{D}$ is

$$
X+\mathcal{D}:=\bigvee_{\mathbf{d} \in \mathcal{D}}(X+\mathbf{d})
$$


Definition 2.3. A multiset family $\mathcal{X}=\left(X_{1}, \ldots, X_{n}\right)$ is a finite vector of multisets $X_{i}$. We call a multiset family $\mathcal{X}=\left(X_{1}, \ldots, X_{n}\right)$ an $n$-multiset family. For $n$-multiset families $\mathcal{X}^{(1)}$ and $\mathcal{X}^{(2)}$ we define

$$
\mathcal{X}^{(1)} \vee \mathcal{X}^{(2)}:=\left(X_{1}^{(1)} \vee X_{1}^{(2)}, \ldots, X_{n}^{(1)} \vee X_{n}^{(2)}\right)
$$

Definition 2.4. A multiset family $\mathcal{X}=\left(X_{1}, \ldots, X_{n}\right)$ is discrete if for each $i$ the multiset $X_{i}$ is discrete, i.e., the underlying set $\underline{X}_{i}$ is discrete and elements in $X_{i}$ have finite multiplicity.

In Section 3 we develop a structure theory for discrete multiset families, which decomposes them into irreducible families. Given an inflation functional equation, let $\psi(\cdot)$ be the inflation operator associated to it, which takes $n$-multiset families to $n$-multiset families, as defined in Section 3.

Definition 2.5. (i) A multiset family $\mathcal{X}=\left(X_{1}, \ldots, X_{n}\right)$ satisfying an inflation functional equation $\psi(\mathcal{X})=\mathcal{X}$ is indecomposable if it cannot be partitioned as $\mathcal{X}=$ $\mathcal{X}^{(1)} \vee \mathcal{X}^{(2)}$ with each $\mathcal{X}^{(i)}$ nonempty such that $\psi\left(\mathcal{X}^{(i)}\right)=\mathcal{X}^{(i)}$ for $i=1$, 2. Otherwise it is decomposable.

(ii) A multiset family $\mathcal{X}=\left(X_{1}, \ldots, X_{n}\right)$ satisfying an inflation functional equation $\psi(\mathcal{X})=\mathcal{X}$ is irreducible if there does not exist a nonempty $\mathcal{X}^{\prime}=\left(X_{1}^{\prime}, \ldots, X_{n}^{\prime}\right)$ satisfying $\psi\left(\mathcal{X}^{\prime}\right)=\mathcal{X}^{\prime}$ with $\mathcal{X}^{\prime} \subsetneq \mathcal{X}$ in the sense that $X_{i}^{\prime} \subseteq X_{i}$ for all $i$, with some $X_{i}^{\prime} \neq X_{i}$. Otherwise it is reducible.

The concepts of indecomposability and irreducibility are equivalent for a multiset family (with a fixed inflation functional equation). If $\mathcal{X}$ is irreducible, then it is necessarily indecomposable. Conversely, if $\mathcal{X}$ is reducible, with $\mathcal{X}^{\prime} \subsetneq \mathcal{X}$, and $\psi\left(\mathcal{X}^{\prime}\right)=\mathcal{X}^{\prime}$, then $\mathcal{X}^{\prime \prime}=\mathcal{X}-\mathcal{X}^{\prime}$ (subtraction done on multiplicity functions) is a multiset family with $\mathcal{X}=\mathcal{X}^{\prime} \vee \mathcal{X}^{\prime \prime}$, and linearity of the inflation functional equation yields $\psi\left(\mathcal{X}^{\prime \prime}\right)=\mathcal{X}^{\prime \prime}$, so that $\mathcal{X}$ is decomposable.

Theorem 2.1 (Decomposition Theorem). Let $\mathcal{X}$ be a multiset family $\mathcal{X}$ that is discrete and satisfies an inflation functional equation $\psi(\mathcal{X})=\mathcal{X}$. Then $\mathcal{X}$ uniquely partitions into a finite number of irreducible discrete multiset families that satisfy the same inflation functional equation.

An inflation functional equation $\psi(\mathcal{X})=\mathcal{X}$ may not have a discrete solution, see Example 8.4. In Section 3 we characterize indecomposable discrete multiset families $\mathcal{X}$ satisfying inflation functional equations, as follows.

Theorem 2.2. Let $\mathcal{X}$ be a multiset family which satisfies an inflation functional equation $\psi(\mathcal{X})=\mathcal{X}$ and is discrete and indecomposable. Then $\mathcal{X}$ is irreducible and is generated by a finite "seed" $\mathcal{S}^{(0)}=\left(S_{1}, S_{2}, \ldots, S_{n}\right)$, which consists of a periodic cycle 
$Y=\left\{\left(\mathbf{x}_{j}, i_{j}\right): 1 \leq j \leq p\right\}$ in which $\mathbf{x}_{j} \in X_{i_{j}}$ and there is some $\mathbf{d}_{j} \in \mathcal{D}_{i_{j+1}, i_{j}}$ with $\mathbf{x}_{j+1}=\mathbf{A} \mathbf{x}_{j}+\mathbf{d}_{j}$, and $\left(\mathbf{x}_{p+1}, i_{p+1}\right)=\left(\mathbf{x}_{1}, i_{1}\right)$. That is,

$$
\mathcal{X}=\lim _{N \rightarrow \infty} \psi^{N}\left(\mathcal{S}^{(0)}\right) .
$$

The periodic cycle $Y$ is the only periodic cycle in $\mathcal{X}$ and its elements each have multiplicity one.

This result appears, in a more precise form, as Theorem 3.3. For each $p$ there are only finitely many periodic cycles $Y$ and they can be effectively enumerated. However, not all periodic cycles $Y$ generate irreducible discrete multiset families. We show that there is an algorithmic procedure, which when given any such "seed" as input, has one of three outcomes:

(1) If the generated multiset system $\mathcal{X}$ is discrete and irreducible, the procedure eventually halts and certifies this holds.

(2) If the generated multiset system $\mathcal{X}$ is not irreducible, or if the limit does not exist, the procedure eventually halts and certifies this holds.

(3) If the generated multiset system $\mathcal{X}$ is irreducible and not discrete, the procedure may not halt.

We also prove in Section 3 a dichotomy concerning the multiplicities of elements appearing in the multisets $X_{i}$ in an irreducible discrete multiset family $\mathcal{X}$ satisfying an inflation functional equation having a primitive subdivision matrix: either they are all bounded by the period $p$ of the generating cycle or else they all have unbounded multiplicities. (Theorem 3.5.) We do not know of an effective computational procedure to tell in general which of these alternatives occurs.

In Sections 4-7 we restrict to the case of inflation functional equations having a primitive subdivision matrix. We study Delone set solutions and self-replicating multitilings.

Definition 2.6. (i) A multiset $X$ is weakly uniformly discrete if there is a radius $r>0$ and a finite $m \geq 1$ such that any open ball of radius $r$ contains at most $m$ points of $X$, counting points with multiplicity.

(ii) A multiset $X$ is uniformly discrete if there is a radius $r>0$ such that any open ball of radius $r$ contains at most one point of $X$, counted with multiplicity. Such an $X$ is necessarily an ordinary set.

(iii) A multiset $X$ is relatively dense if there is a finite radius $R>0$ such that each closed ball of radius $R$ contains at least one point of $X$.

Note that a finite union of weakly uniformly discrete sets is weakly uniformly discrete.

Definition 2.7. (i) A multiset $X$ is a weak Delone multiset if it is weakly uniformly discrete and relatively dense.

(ii) A multiset $X$ is a Delone set if it is uniformly discrete and relatively dense. That is, it is an ordinary set which has both a finite nonzero packing radius and a finite covering radius by equal spheres. 
A finite union of Delone sets need not be a Delone set, while a finite union of weak Delone multisets is a weak Delone multiset.

Definition 2.8. (i) A multiset family $\mathcal{X}=\left(X_{1}, \ldots, X_{n}\right)$ is a weak Delone multiset family if $\mathcal{X}$ satisfies the inflation functional equation (1.3) in $\mathbb{R}^{d}$ and each multiset $X_{i}$ is a weak Delone multiset.

(ii) It is a substitution Delone set family if it is a weak substitution Delone multiset family and each $X_{i}$ is a Delone set.

Weak substitution Delone multiset families can sometimes be associated to $p$-thick multiple tilings, for some $p \geq 2$.

In Section 4 we prove the following result.

Theorem 2.3 (Perron Eigenvalue Condition). If the inflation functional equation $\psi(\mathcal{X})=\mathcal{X}$ with a primitive subdivision matrix $\mathrm{S}=\left[\left|\mathcal{D}_{i j}\right|\right]$ has a solution $\mathcal{X}$ that is a weak Delone multiset family, then the Perron eigenvalue $\lambda(\mathrm{S})$ of $\mathrm{S}$ satisfies

$$
\lambda(\mathrm{S})=|\operatorname{det}(\mathrm{A})|
$$

The narrowest class of solutions to the inflation functional equation are those having the following tiling property.

Definition 2.9. A multiset family $\mathcal{X}=\left(X_{1}, \ldots, X_{n}\right)$ is called a self-replicating multitiling family for a given system $\left(A, \mathcal{D}_{i j}\right)$ in $(1.3)$ with a primitive subdivision matrix $S$ if:

(i) $\mathcal{X}$ is a substitution Delone set family for $\left(\mathrm{A}, \mathcal{D}_{i j}\right)$.

(ii) The associated multi-tile equation (1.1) has a unique solution $\mathcal{T}:=\left(T_{1}, \ldots, T_{n}\right)$ with each $T_{i}$ of positive Lebesgue measure.

(iii) The sets $\left\{T_{i}+\mathbf{x}_{i}: 1 \leq i \leq n\right.$ and $\left.\mathbf{x}_{i} \in X_{i}\right\}$ tile $\mathbb{R}^{d}$.

This definition requires that the multiset family $\mathcal{X}$ give a 1-thick tiling, rather than a $p$-thick multiple tiling for some $p \geq 2$, or a $p$-packing for some $p \geq 1$ that is not a tiling.

In Section 5 we study the multi-tiling functional equation and show that a necessary condition for a solution with some $T_{i}$ having positive Lebesgue measure is that $\lambda(\mathrm{S}) \geq$ $|\operatorname{det}(\mathrm{A})|$. Assuming that the Perron eigenvalue condition $\lambda(\mathrm{S})=|\operatorname{det}(\mathrm{A})|$ holds, in Theorem 5.5 we characterize when solutions of positive Lebesgue measure exist.

In Section 6 we prove the following result, which shows that substitution Delone set families are related to the existence of self-replicating multi-tilings. In this result $\psi^{N}(\cdot)$ denotes the inflation operator $\psi(\cdot)$ composed with itself $N$ times, whose associated inflation matrix is $\mathrm{A}^{N}$.

Theorem 2.4. Let $\psi(\mathcal{X})=\mathcal{X}$ be an inflation functional equation that has a primitive subdivision matrix. Then the following conditions are equivalent:

(i) For some $N>0$ there exists a weak Delone multiset family $\hat{\mathcal{X}}$ satsfying $\psi^{N}(\hat{\mathcal{X}})=\hat{\mathcal{X}}$. 
(ii) For some $N>0$ there exists a self-replicating multi-tiling family $\hat{\mathcal{X}}$ for the inflation functional equation $\psi^{N}(\cdot)$.

(iii) The subdivision matrix $\mathrm{S}$ satisfies the Perron eigenvalue condition $\lambda(\mathrm{S})=|\operatorname{det}(\mathrm{A})|$ and the unique compact solution $\left(T_{1}, \ldots, T_{n}\right)$ of the associated multi-tile functional equation consists of sets $T_{i}$ that have positive Lebesgue measure, $1 \leq i \leq n$, and each $T_{i}$ is the closure of its interior $T_{i}=\overline{T_{i}^{\circ}}$.

These conditions imply that the Perron eigenvalue condition holds. Theorem 6.1 shows that another equivalent condition is that the Perron eigenvalue condition holds and there is a weakly uniformly discrete multiset family $\hat{\mathcal{X}}$ satisfying $\psi^{N}(\hat{\mathcal{X}})=\hat{\mathcal{X}}$ for some $N$. It is known there are many restrictions on the data $\left(\mathrm{A}, \mathcal{D}_{i j}\right)$ in order for the conditions of Theorem 2.4 to hold. A number of different necessary (resp. sufficient) conditions on the data $\left(\mathrm{A}, \mathcal{D}_{i j}\right)$ are given in [8]-[11], [18]-[20], [29], [30], and [35]-[37].

In Section 7 we give a sufficient condition for a substitution Delone set family to be a self-replicating multi-tiling (Theorem 7.1). In Section 8 we give examples and counterexamples showing the limits of our results.

\section{Structure of Discrete Multiset Solutions}

We consider multiset families $\mathcal{X}=\left(X_{1}, \ldots, X_{n}\right)$ satisfying the inflation functional equation

$$
X_{i}=\bigvee_{j=1}^{n}\left(\mathrm{~A}\left(X_{j}\right)+\mathcal{D}_{i j}\right), \quad 1 \leq i \leq n
$$

The inflation operator $\psi(\cdot)$ associated to data $\left(\mathrm{A}, \mathcal{D}_{i j}\right)$ is an operator which maps arbitrary $n$-multiset families $\mathcal{X}$ to $n$-multiset families $\psi(\mathcal{X})=\mathcal{X}^{\prime}$ as follows. Given $\mathcal{X}$ (not necessarily satisfying (3.1)), we define $\psi(\mathcal{X})=\left(X_{1}^{\prime}, \ldots, X_{n}^{\prime}\right)$ by

$$
X_{i}^{\prime}:=\bigvee_{j=1}^{n}\left(\mathrm{~A}\left(X_{j}\right)+\mathcal{D}_{i j}\right), \quad 1 \leq i \leq n
$$

The inflation functional equation (3.1) then asserts that $\mathcal{X}$ is a fixed point of $\psi(\cdot)$, i.e., $\psi(\mathcal{X})=\mathcal{X}$.

In this section we determine the structure of multiset families $\mathcal{X}$ that are discrete and satisfy $\psi(\mathcal{X})=\mathcal{X}$, where we put no restriction on $\psi(\cdot)$. The results allow imprimitive substitution matrices, unless otherwise stated. We describe the structure of such sets that are also irreducible (Theorems 3.3 and 3.5). We show that every multiset family $\mathcal{X}$ that is discrete and satisfies $\psi(\mathcal{X})=\mathcal{X}$ uniquely partitions into a finite number of irreducible sets (Theorem 2.1).

We consider solutions to the inflation functional equation (3.1) built up by an iterative process starting from a finite multiset which is a "seed." Let

$$
\mathcal{S}^{(0)}:=\left(S_{1}^{(0)}, \ldots, S_{n}^{(0)}\right)
$$


be a system of finite multisets, and iteratively define the finite multiset family $\mathcal{S}^{(k+1)}$ by $\mathcal{S}^{(k+1)}=\psi\left(\mathcal{S}^{(k)}\right)$, i.e.,

$$
\mathcal{S}_{i}^{(k+1)}:=\bigvee_{j=1}^{n}\left(\mathrm{~A}\left(S_{j}^{(k)}\right)+\mathcal{D}_{i j}\right), \quad 1 \leq i \leq n .
$$

We say that $\mathcal{S}^{(0)}$ satisfies the inclusion property $\mathcal{S}^{(0)} \subseteq \mathcal{S}^{(1)}$ if

$$
S_{i}^{(0)} \subseteq S_{i}^{(1)} \quad \text { for } \quad 1 \leq i \leq n,
$$

in the sense of multisets.

Lemma 3.1. If a multiset family $\mathcal{S}^{(0)}$ is finite and satisfies the inclusion condition $\mathcal{S}^{(0)} \subseteq \psi\left(\mathcal{S}^{(0)}\right)$, then

$$
\mathcal{S}^{(k)} \subseteq \mathcal{S}^{(k+1)} \quad \text { for all } \quad k \geq 0 .
$$

The following limit set is well-defined:

$$
\underline{X}_{i}:=\lim _{k \rightarrow \infty} \underline{S}_{i}^{(k)}, \quad 1 \leq i \leq n,
$$

where $\underline{X}_{i}$ is regarded as a countable point set. For each $\mathbf{x} \in \underline{X}_{i}$ the multiplicity function has a limit,

$$
m_{X_{i}}(\mathbf{x}):=\lim _{k \rightarrow \infty} m\left(S_{i}^{(k)}, \mathbf{x}\right),
$$

where this multiplicity may take the value $+\infty$. If all multiplicities in (3.7) remain finite, then the multisets $X_{i}$ are well-defined and the multiset family $\mathcal{X}=\left(X_{1}, \ldots, X_{n}\right)$ satisfies $\psi(\mathcal{X})=\mathcal{X}$.

Remark. If all the multiplicities remain finite, we write $\mathcal{X}:=\lim _{k \rightarrow \infty} \mathcal{S}^{(k)}$. The limit multiset family $\mathcal{X}$ need not be discrete, and the underlying sets could even be dense.

Proof. The inclusion (3.5) follows by induction on $k$, since $\mathcal{S}^{(k-1)} \subseteq \mathcal{S}^{(k)}$ yields

$$
\mathcal{S}^{(k)}=\psi\left(\mathcal{S}^{(k-1)}\right) \subseteq \psi\left(\mathcal{S}^{(k)}\right)=\mathcal{S}^{(k+1)} .
$$

The other statements follow easily from (3.5).

To analyze further multiset families $\mathcal{X}$ that satisfy $\psi(\mathcal{X})=\mathcal{X}$, we put the structure of a colored directed graph on $\mathcal{X}$. The vertices of this graph are the points in the disjoint union of the underlying sets $\underline{X}_{i}$, with vertices being labeled $(\mathbf{x}, i)$, where $\mathbf{x} \in \underline{X}_{i} \in \mathbb{R}^{d}$ and $i$ is the "color." For each $\mathbf{x}_{j} \in X_{j}$ and each $\mathbf{d}_{j} \in \mathcal{D}_{i j}$, set

$$
\mathbf{x}_{i}^{\prime}:=A \mathbf{x}_{j}+\mathbf{d}_{j} \in X_{i},
$$

and put a directed edge $\mathbf{x}_{j} \rightarrow \mathbf{x}_{i}^{\prime}$ in the graph. We call $\mathbf{x}_{i}^{\prime}$ an offspring of $\mathbf{x}_{j}$, and call $\mathbf{x}_{j}$ a preimage of $\mathbf{x}_{i}^{\prime}$. We denote this (infinite) colored directed graph by $\mathcal{G}(\mathcal{X})$. We also assign to each vertex $\mathbf{x} \in \underline{X}_{i}$ a weight which is its multiplicity $m_{X_{i}}(\mathbf{x})$. 
Lemma 3.2. Let $\mathcal{X}=\left(X_{1}, \ldots, X_{n}\right)$ be a multiset family which is discrete and satisfies the inflation functional equation $\psi(\mathcal{X})=\mathcal{X}$.

(i) There is a finite multiset family $\mathcal{S}^{(0)} \subseteq \mathcal{X}$ with the inclusion property, such that

$$
\mathcal{X}=\lim _{k \rightarrow \infty} \mathcal{S}^{(k)} .
$$

(ii) The directed graph $\mathcal{G}(\mathcal{X})$ contains a directed cycle.

Proof. (i) We first show that there exists a radius $R^{\prime}>0$, depending only on $\psi(\cdot)$, such that every vertex in $\mathcal{X}$ can be reached by a directed path in the graph $\mathcal{G}(\mathcal{X})$ from some point $\mathbf{x} \in \mathcal{X} \cap B_{R^{\prime}}(\mathbf{0})$, where $B_{R^{\prime}}(\mathbf{0}):=\left\{\mathbf{x} \in \mathbb{R}^{d}:\|\mathbf{x}\| \leq R^{\prime}\right\}$. To show this, we set

$$
\lambda\left(A^{-1}\right)=\min \left\{|\lambda|: \lambda \text { is an eigenvalue of } A^{-1}\right\},
$$

and $\lambda\left(A^{-1}\right)<1$ since $A$ is expanding. Fix a $\rho>1$ such that $\lambda\left(A^{-1}\right)<1 / \rho<1$. It is shown in [23] that there exists a norm $\|\cdot\|_{\mathrm{A}}$ on $\mathbb{R}^{d}$ with the property that

$$
\left\|A^{-1} \mathbf{x}\right\|_{\mathrm{A}} \leq \frac{1}{\rho}\|\mathbf{x}\|_{\mathrm{A}} \quad \text { for all } \quad \mathbf{x} \in \mathbb{R}^{d} .
$$

The norm is defined by $\|\mathbf{x}\|_{\mathrm{A}}:=\sum_{k=1}^{\infty} \rho^{k}\left\|A^{-k} \mathbf{x}\right\|$.

We claim that there exists an $R>0$ such that, for any vertex $\mathbf{x}_{i}^{\prime} \in \underline{X}_{i}$ with $\left\|\mathbf{x}_{i}^{\prime}\right\|_{\mathrm{A}} \geq R$, any preimage $\mathbf{x}_{j} \in \underline{X}_{j}$ of $\mathbf{x}_{i}^{\prime}$ must satisfy

$$
\left\|\mathbf{x}_{j}\right\|_{\mathrm{A}} \leq \frac{2}{1+\rho}\left\|\mathbf{x}_{i}^{\prime}\right\|_{\mathrm{A}}
$$

To see this, let

$$
C:=\max \left\{\left\|\mathbf{d}_{j}\right\|_{\mathrm{A}}: \mathbf{d}_{j} \in \mathcal{D}_{i j}, 1 \leq i, j \leq n\right\} \quad \text { and } \quad R:=\frac{\rho+1}{\rho-1} C .
$$

It follows from $\mathbf{x}_{j}=\mathrm{A}^{-1}\left(\mathbf{x}_{i}^{\prime}-\mathbf{d}_{j}\right)$ that

$$
\left\|\mathbf{x}_{j}\right\|_{\mathrm{A}} \leq \frac{1}{\rho}\left(\left\|\mathbf{x}_{i}^{\prime}\right\|_{\mathrm{A}}+C\right) \leq \frac{2}{1+\rho}\left\|\mathbf{x}_{i}^{\prime}\right\|_{\mathrm{A}} .
$$

Thus if a vertex $\mathbf{x}$ has $\|\mathbf{x}\|_{\mathrm{A}} \geq R$, then every preimage of $\mathbf{x}$ in the graph $\mathcal{G}(\mathcal{X})$ is smaller in the norm $\|\cdot\|_{\mathrm{A}}$ by a multiplicative constant $2 /(1+\rho)<1$. It follows that every vertex $\mathbf{x}$ in $\mathcal{G}(\mathcal{X})$ can be reached by a finite directed path in $\mathcal{G}(\mathcal{X})$ starting from some vertex $\mathbf{x}^{\prime}$ with $\left\|\mathbf{x}^{\prime}\right\|_{\mathrm{A}} \leq R$. Since the set

$$
\tilde{B}_{R}:=\left\{\mathbf{y} \in \mathbb{R}^{d}:\|\mathbf{y}\|_{\mathrm{A}} \leq R\right\}
$$

is compact, it is contained in some ball of radius $R^{\prime}$ around $\mathbf{0}$, which gives the result.

We now take the multiset family $\mathcal{S}^{(0)}=\left(S_{1}^{(0)}, \ldots, S_{n}^{(0)}\right)$ such that $S_{i}$ consists of those elements $\mathbf{x}$ of $X_{i}$ with $\|\mathbf{x}\|_{\mathrm{A}} \leq R$, counting multiplicities, i.e., we take

$$
m_{S_{i}^{(0)}}(\mathbf{x}):=m_{X_{i}}(\mathbf{x}) \quad \text { if } \quad \mathbf{x} \in \underline{S}_{i}^{(0)} .
$$


The multiset family $\mathcal{S}^{(0)}$ is a finite family since $\mathcal{X}$ is discrete. The inclusion property

$$
\mathcal{S}^{(0)} \subseteq \mathcal{S}^{(1)}=\psi\left(\mathcal{S}^{(0)}\right)
$$

holds because all preimages of vertices of $\mathcal{G}(\mathcal{X})$ in $\left\{\mathbf{x} \in \mathbb{R}^{d}:\|\mathbf{x}\|_{\mathrm{A}} \leq R\right\}$ lie in this set, since $\left\|\mathbf{x}_{j}\right\|_{\mathrm{A}} \leq(1 / \rho)(R+C) \leq R$. Since $\mathcal{S}^{(0)} \subseteq \mathcal{X}$ and $\psi(\mathcal{X})=\mathcal{X}$ we obtain $\mathcal{S}^{(k)} \subseteq \mathcal{X}$ for all $k$, viewed as multisets, so that

$$
\mathcal{X}^{\prime}:=\lim _{k \rightarrow \infty} \mathcal{S}^{(k)}
$$

exists and is a multiset. Now $\mathcal{S}^{(0)}$ contains all points $\mathbf{x}$ of $\mathcal{X}$ with the correct multiplicities for $\|\mathbf{x}\|_{\mathrm{A}} \leq R$. Now, by induction on $k \geq 0$, one proves:

For all $\mathbf{x}$ with $\|\mathbf{x}\|_{\mathrm{A}} \leq((1+\rho) / 2)^{k} R$, one has for $1 \leq i \leq n$ that the multiplicity

$$
m\left(\mathcal{S}_{i}^{(k)}, \mathbf{x}\right)=m_{X_{i}}(\mathbf{x}) .
$$

The base case $k=0$ holds by construction, and the induction step follows because all preimages of a vertex in $\left\{\|\mathbf{x}\| \leq((1+\rho) / 2)^{k} R\right\}$ lie in $\left\{\|\mathbf{x}\| \leq((1+\rho) / 2)^{k-1} R\right\}$. We conclude that $\mathcal{X}^{\prime}=\mathcal{X}$, which gives (i).

(ii) We choose $R$ as in (i). Pick a point $\mathbf{x}$ in $\mathcal{X}$ with

$$
\mathbf{x} \in \tilde{B}_{R}:=\left\{\mathbf{y} \in \mathbb{R}^{d}:\|\mathbf{y}\|_{\mathrm{A}} \leq R\right\},
$$

which must exist by the argument in (i). All preimages of $\mathbf{x}$ necessarily lie in $\tilde{B}_{R}$. Since $\mathcal{X}$ is discrete, if we follow a chain of successive preimages of a vertex $\mathbf{x}$ in $\mathcal{G}(\mathcal{X})$ we stay in the finite set $\mathcal{X} \cap \tilde{B}_{R}$, so some vertex must occur twice. The path from this vertex to itself forms a directed cycle in $\mathcal{G}(\mathcal{X})$.

Recall that a multiset family $\mathcal{X}$ satisfying an inflation functional equation is indecomposable if it has no nontrivial partition $\mathcal{X}=\mathcal{X}_{1} \vee \mathcal{X}_{2}$, with both $\mathcal{X}_{i}$ satisfying the same functional equation, and it is irreducible if there is no nonempty $\mathcal{X}^{\prime} \subsetneq \mathcal{X}$ satisfying the same functional equation.

Theorem 3.3 (Irreducible Set Characterization). Suppose that the multiset family $\mathcal{X}$ is discrete and satisfies an inflation functional equation $\psi(\mathcal{X})=\mathcal{X}$. The following are equivalent:

(i) $\mathcal{X}$ is indecomposable.

(ii) $\mathcal{X}$ is irreducible.

(iii) The graph $\mathcal{G}(\mathcal{X})$ contains exactly one directed cycle (which may be a loop), and the elements of this cycle have multiplicity one.

If condition (iii) holds, let $Y=\left\{\mathbf{x}_{1}, \ldots, \mathbf{x}_{p}\right\}$ be the cycle in $\mathcal{G}(\mathcal{X})$ with multiplicity one and define the multiset family $\mathcal{S}^{(0)}=\left(S_{1}^{(0)}, \ldots, S_{n}^{(0)}\right)$ by $S_{i}=\{\mathbf{x} \in Y: \mathbf{x}$ has color $i\}$. Then $\mathcal{S}^{(0)} \subseteq \psi\left(\mathcal{S}^{(0)}\right)$ and

$$
\mathcal{X}=\lim _{k \rightarrow \infty} \mathcal{S}^{(k)}
$$


Remark. In view of (iii) and (3.14) we call $\mathcal{S}^{(0)}$ the generating cycle of the irreducible multiset system $\mathcal{X}$, and we call $p$ the period of this cycle.

Proof. We show (i) $\Leftrightarrow$ (ii) $\Rightarrow$ (iii) $\Rightarrow$ (i). Just before stating Theorem 2.1 we showed that implication (i) $\Leftrightarrow$ (ii) holds for all multiset solutions to a fixed inflation functional equation.

(ii) $\Rightarrow$ (iii) Lemma 3.2 shows that $\mathcal{X}$ is generated by the points $\mathbf{x}$ in $\mathcal{X}$ that lie in the compact region $\|\mathbf{x}\|_{\mathrm{A}} \leq R$, and that $\mathcal{G}(\mathcal{X})$ contains vertices forming a directed cycle $Y$ inside this region. The vertices of $Y$ define a finite multiset family $\mathcal{S}^{(0)}=\left(S_{1}^{(0)}, \ldots, S_{n}^{(0)}\right)$, with $S_{i}^{(0)}=\underline{X}_{i} \cap Y$, and all elements of $S_{i}^{(0)}$ have multiplicity one. It is clear that $\mathcal{S}^{(0)} \subseteq \mathcal{S}^{(1)}=\psi\left(\mathcal{S}^{(0)}\right)$ since each element of $\mathcal{S}^{(0)}$ has a preimage in $\mathcal{S}^{(0)}$. Since each $\mathcal{S}^{(k)} \subseteq \mathcal{X}$ the limit

$$
\mathcal{X}^{\prime}:=\lim _{k \rightarrow \infty} \mathcal{S}^{(k)}
$$

exists and is a multiset family with $\mathcal{X}^{\prime} \subseteq \mathcal{X}$. We have $\mathcal{X}^{\prime}=\mathcal{X}$, for if not, this contradicts the irreducibility of $\mathcal{X}$.

We next show that $\mathcal{X}$ has multiplicity one on the vertices of $Y$. Suppose not. The elements of $\mathcal{S}^{(0)}$ have multiplicity one, and at some later stage some vertex $(\mathbf{x}, i)$ of the cycle $Y$ has multiplicity exceeding one in some $\mathcal{S}^{(k)}$. Thus there exists a path to $(\mathbf{x}, i)$ of length $k$ arising from some vertex $(\mathbf{y}, j)$ in $\mathcal{S}^{(0)}$, which does not arise purely from moving on the cycle $Y$. Since the vertex $(\mathbf{y}, j)$ can be reached from $(\mathbf{x}, i)$ by moving around the cycle $Y$, taking $l$ steps, say, we obtain a directed path from $(\mathbf{x}, i)$ to itself of length $k+l$ which does not stay in the cycle $Y$. Now we obtain two distinct directed paths of length $(k+l) p$ from $(\mathbf{x}, i)$ to itself, one by wrapping around the cycle $Y k+l$ times, the other by repeating the path of length $k+l$ exactly $p$ times. Since we can concatenate these two distinct paths in any order, it follows that the multiplicity of $(\mathbf{x}, i)$ in $\mathcal{S}^{((k+l) p m)}$ is at least $2^{m}$. This implies that the vertex $(\mathbf{x}, i)$ has unbounded multiplicity as $m \rightarrow \infty$, so that $\lim _{k \rightarrow \infty} \mathcal{S}^{(k)}$ does not exist, a contradiction. Thus $\mathcal{X}$ has multiplicity one on the cycle $Y$.

Finally we show that $\mathcal{X}$ contains only one directed cycle. Suppose not, and that $\mathcal{G}(\mathcal{X})$ contains a directed cycle $Y^{\prime}$ different from $Y$. The same argument as above says that $\mathcal{X}$ is generated by the cycle $Y^{\prime}$ and that all elements of $Y^{\prime}$ in $\mathcal{X}$ have multiplicity one. By exchanging $Y$ and $Y^{\prime}$ if necessary we may suppose that $Y$ has a vertex not contained in $Y^{\prime}$. Now following a path from a vertex in $Y \backslash Y^{\prime}$ to $Y^{\prime}$ we see that there exists an $\mathbf{x}^{\prime} \in Y^{\prime}$ that has a preimage not in $Y^{\prime}$. However, $\mathbf{x}^{\prime}$ also has a preimage in $Y^{\prime}$ because $Y^{\prime}$ is a cycle. This implies that the multiplicity of $\mathbf{x}^{\prime}$ is at least two, a contradiction. Thus $\mathcal{G}(\mathcal{X})$ contains exactly one directed cycle.

(iii) $\Rightarrow$ (i) Suppose that $\mathcal{G}(\mathcal{X})$ contains exactly one directed cycle $Y$, of multiplicity one. We argue by contradiction. Suppose that $\mathcal{X}$ were not indecomposable. Then we can write $\mathcal{X}=\mathcal{X}^{\prime} \vee \mathcal{X}^{\prime \prime}$, where both $\mathcal{X}^{\prime}$ and $\mathcal{X}^{\prime \prime}$ satisfy the inflation functional equation. Now Lemma 3.2(ii) implies that both $\mathcal{G}\left(\mathcal{X}^{\prime}\right)$ and $\mathcal{G}\left(\mathcal{X}^{\prime \prime}\right)$ contain a directed cycle. Therefore $\mathcal{G}(\mathcal{X})$ either contains two directed cycles or else contains a directed cycle of multiplicity at least two. This contradicts the hypothesis. 
We can now prove the Decomposition Theorem.

Proof of Decomposition Theorem 2.1. If $\mathcal{X}$ is not irreducible, then it is decomposable and we have $\mathcal{X}=\mathcal{X}^{(1)} \vee \mathcal{X}^{(2)}$ where each $\mathcal{X}^{(i)}$ satisfies $\mathcal{X}^{(i)}=\psi\left(\mathcal{X}^{(i)}\right)$. Lemma 3.2 showed that $\mathcal{X}$ and $\mathcal{X}^{(i)}$ are generated by elements in the bounded region $\|\mathbf{x}\|_{\mathrm{A}} \leq$ $R$. So the total multiplicity of elements of each $\mathcal{X}^{(i)}$ in the region $\|\mathbf{x}\|_{\mathrm{A}} \leq R$ is strictly smaller than that of elements of $\mathcal{X}$ in the region $\|\mathbf{x}\|_{\mathrm{A}} \leq R$. If one of $\mathcal{X}^{(i)}$ is not irreducible, then we can further partition it, and decrease the total multiplicity in $\|\mathbf{x}\|_{\mathrm{A}} \leq R$ again. Since $\mathcal{X}$ is discrete, this process will end in finitely many steps, yielding

$$
\mathcal{X}=\bigvee_{i=1}^{N} \mathcal{X}^{(i)}
$$

To see that the partition is unique, to each element $\mathcal{X}_{i}$ of the partition is associated a unique directed cycle in $\mathcal{G}(\mathcal{X})$ of multiplicity one. However, each directed cycle in $\mathcal{G}(\mathcal{X})$, counted with multiplicity one, must appear in some element of each partition.

Another consequence of Theorem 3.3 is a finiteness condition on discrete irreducible multiset families.

Lemma 3.4. Let $\psi(\cdot)$ be an inflation functional equation with data $\left(\mathrm{A}, \mathcal{D}_{i j}\right)$. Then for each $p \geq 1$ there are at most finitely many discrete multiset families (possibly none) $\mathcal{X}$ satisfying $\psi(\mathcal{X})=\mathcal{X}$ that are irreducible and have a periodic cycle in $\mathcal{G}(\mathcal{X})$ of minimal period $p$.

Proof. Any periodic cycle $\left\{\left(\mathbf{x}_{0}, i_{0}\right),\left(\mathbf{x}_{1}, i_{1}\right), \ldots,\left(\mathbf{x}_{p-1}, i_{p-1}\right)\right\}$ constructed in Theorem 3.3 has the form

$$
\mathbf{x}_{k}=\mathrm{A} \mathbf{x}_{k-1}+\mathbf{d}_{k}, \quad 1 \leq k \leq p,
$$

with $\mathbf{d}_{k} \in \mathcal{D}_{i_{k}, i_{k-1}}$ and $\left(\mathbf{x}_{p}, i_{p}\right)=\left(\mathbf{x}_{0}, i_{0}\right)$. It follows that

$$
\mathbf{x}_{0}=\mathrm{A}^{p} \mathbf{x}_{0}+\sum_{k=1}^{p} \mathrm{~A}^{p-k} \mathbf{d}_{k} .
$$

Thus

$$
\mathbf{x}_{0}=-\left(\mathrm{A}^{p}-\mathrm{I}\right)^{-1}\left(\sum_{k=1}^{p} \mathrm{~A}^{p-k} \mathbf{d}_{k}\right),
$$

where the matrix $A^{p}-I$ is invertible since $A$ is expanding. There are only a finite set of choices for the digits $\left\{\mathbf{d}_{1}, \ldots, \mathbf{d}_{p}\right\}$, so the number of choices for $\mathbf{x}_{0}$ is finite.

We continue the study of discrete multiset families $\mathcal{X}$ with $\psi(\mathcal{X})=\mathcal{X}$ that are irreducible. We obtain further information on the multiplicities that occur in such $\mathcal{X}$, under the extra hypothesis that the subdivision matrix is primitive. 
Theorem 3.5 (Multiplicity Dichotomy). Suppose that the multiset family $\mathcal{X}$ is discrete and satisfies the inflation functional equation $\psi(\mathcal{X})=\mathcal{X}$ with a primitive subdivision matrix. If $\mathcal{X}=\left(X_{1}, X_{2}, \ldots, X_{n}\right)$ is indecomposable, then exactly one of the following cases holds:

(i) Every element of each multiset $X_{i}$ has multiplicity at most $p$, the period of the generating cycle in the directed graph $\mathcal{G}(\mathcal{X})$.

(ii) For each multiset $X_{i}$ in $\mathcal{X}$, the multiplicities $m_{X_{i}}(\mathbf{x})$ of $\mathbf{x} \in X_{i}$ are unbounded.

Remarks. (1) For the general case of imprimitive $S$, one can prove a weaker multiplicity dichotomy; it applies only to those $X_{i}$ such that $i$ lies in the strongly connected component of the underlying graph of $S$ that contains the generating cycle.

(2) Both cases of the Multiplicity Dichotomy occur. Example 8.5 gives an occurrence of case (i) in which all $X_{i}$ have bounded multiplicity, with some multiplicities exceeding one. Example 8.1 exhibits case (ii).

Proof. The multiplicity of a vertex $(\mathbf{x}, i)$ in the graph $\mathcal{G}(\mathcal{X})$ is $m(\mathbf{x}, i):=m_{X_{i}}(\mathbf{x})$. Fix a point $\left(\mathbf{x}_{0}, i_{0}\right)$ in the generating cycle. We claim: For any point $(\mathbf{x}, i) \neq\left(\mathbf{x}_{0}, i_{0}\right)$ in $\mathcal{X}$ the multiplicity $m(\mathbf{x}, i)$ is equal to the number of cycle-free directed paths from $\left(\mathbf{x}_{0}, i_{0}\right)$ to $(\mathbf{x}, i)$. This is proved by induction on the length $k$ of the longest cycle-free directed path from $\mathbf{x}_{0}$ to $\mathbf{x}$, which is bounded by (3.12). The base case $k=1$ is immediate. We have

$$
m(\mathbf{x}, i)=\sum_{\left(\mathbf{x}^{\prime}, j\right) \in P(\mathbf{x}, i)} m\left(\mathbf{x}^{\prime}, j\right),
$$

where $P(\mathbf{x}, i)$ is the set of preimages of $(\mathbf{x}, i)$ in $\mathcal{G}(\mathcal{X})$. Assuming $(\mathbf{x}, i) \neq\left(\mathbf{x}_{0}, i_{0}\right)$ all preimages have a shorter longest cycle-free path, and the induction step follows.

Now assume that some vertex $\left(\mathbf{x}_{1}, j\right)$ has multiplicity $m\left(\mathbf{x}_{1}, j\right)=q>p$. Without loss of generality we may assume that $\left(\mathbf{x}_{1}, j\right)$ and $\left(\mathbf{x}_{0}, i_{0}\right)$ have the same color $j=i_{0}$, for if not by the primitivity hypothesis we can always find a descendant $\left(\mathbf{x}_{1}^{\prime}, i_{0}\right)$ of $\left(\mathbf{x}_{1}, j\right)$, and clearly $m\left(\mathbf{x}_{1}^{\prime}, i_{0}\right) \geq m\left(\mathbf{x}_{1}, j\right)>p$. By the pigeonhole principle there exist two cyclefree directed paths from $\left(\mathbf{x}_{0}, i_{0}\right)$ to $\left(\mathbf{x}_{1}, i_{0}\right)$, whose lengths are $L$ and $L^{\prime}$ respectively with $L \equiv L^{\prime}(\bmod p)$. Say $L^{\prime}=L+s p$ for some $s \geq 0$. We can then create a second directed path of length $L^{\prime}$ by first going around the cycle $s$ times in the beginning and then following the path of length $L$. We show that these two different directed paths force the multiplicity of vertices to be unbounded.

Since each directed edge can be labeled by elements in $\mathcal{D}_{i j}, 1 \leq i, j \leq m$, we label the two directed paths by

$$
\mathcal{P}_{1}=\left(\mathbf{d}_{1}, \ldots, \mathbf{d}_{L}\right), \quad \mathcal{P}_{2}=\left(\mathbf{d}_{1}^{\prime}, \ldots, \mathbf{d}_{L}^{\prime}\right) .
$$

The fact that $\mathbf{x}_{0}$ and $\mathbf{x}_{1}$ have the same color $i$ implies that $\mathbf{d}_{1} \in \mathcal{D}_{j i}, \mathbf{d}_{L} \in \mathcal{D}_{i k}$ and $\mathbf{d}_{1}^{\prime} \in \mathcal{D}_{j^{\prime}}, \mathbf{d}_{L}^{\prime} \in \mathcal{D}_{i k^{\prime}}$ for some $j, k, j^{\prime}, k^{\prime}$. Evaluating the two paths yields

$$
\mathbf{x}_{1}=\mathrm{A}^{L} \mathbf{x}_{0}+\sum_{j=1}^{L} \mathrm{~A}^{j-1} \mathbf{d}_{j}=\mathrm{A}^{L} \mathbf{x}_{0}+\sum_{j=1}^{L} \mathrm{~A}^{j-1} \mathbf{d}_{j}^{\prime} .
$$


Thus $\sum_{j=1}^{L} \mathrm{~A}^{j-1} \mathbf{d}_{j}=\sum_{j=1}^{L} \mathrm{~A}^{j-1} \mathbf{d}_{j}^{\prime}$. This means that following the two paths $\mathcal{P}_{1}$ and $\mathcal{P}_{2}$ from $\left(\mathbf{x}_{1}, i_{0}\right)$ will lead to the same vertex $\left(\mathbf{x}_{2}, i_{0}\right)$, which also has color $i_{0}$. This process can be continued to obtain vertices $\left(\mathbf{x}_{k}, i_{0}\right), k \geq 1$, all of which have the same color $i_{0}$. Note that there are at least $2^{k}$ distinct directed paths from $\left(\mathbf{x}_{0}, i_{0}\right)$ to $\left(\mathbf{x}_{k}, i_{0}\right)$ as we may concatenate $\mathcal{P}_{1}$ and $\mathcal{P}_{2}$ in any combination. These $2^{k}$ directed paths remain distinct after removing the cycles in the initial segment, hence $m\left(\mathbf{x}_{k}, i_{0}\right) \geq 2^{k}$. Thus the multiplicity of $X_{i_{0}}$ is unbounded. Now the primitivity of the subdivision matrix implies that any vertex $\left(\mathbf{x}, i_{0}\right)$ has a descendant in each $X_{i}$. It follows that the multiplicity function is unbounded on every $X_{i}$.

As we have shown, all irreducible discrete multiset families $\mathcal{X}$ are generated iteratively from a cycle as "seed." However, the multiset family generated from a given cycle need not be discrete. The following lemma gives a criterion for discreteness.

Lemma 3.6. Let $\psi(\cdot)$ be an inflation functional equation with data $\left(\mathrm{A}, \mathcal{D}_{i j}\right)$, and let $\mathcal{S}^{(0)}$ be a finite multiset family with the inclusion property $\mathcal{S}^{(0)} \subseteq \mathcal{S}^{(1)}=\psi\left(\mathcal{S}^{(0)}\right)$. Set $R=R(\mathrm{~A}, \mathcal{D})$ equal to the constant in (3.11). If, for some $k \geq 1$,

$$
\mathcal{S}^{(k)} \cap\left\{\mathbf{x} \in \mathbb{R}^{d}:\|\mathbf{x}\|_{\mathrm{A}} \leq R\right\}=\mathcal{S}^{(k+1)} \cap\left\{\mathbf{x} \in \mathbb{R}^{d}:\|\mathbf{x}\|_{\mathrm{A}} \leq R\right\}
$$

counting multiplicities, then the limit

$$
\mathcal{X}:=\lim _{k \rightarrow \infty} \mathcal{S}^{(k)}
$$

is a discrete multiset family that satisfies $\psi(\mathcal{X})=\mathcal{X}$. Conversely, if $\mathcal{X}$ is discrete, then (3.18) holds for all sufficiently large $k$.

Proof. The iterative scheme $\mathcal{S}^{(k+1)}=\psi\left(\mathcal{S}^{(k)}\right)$ is geometrically expanding outside the set $\left\{\mathbf{x} \in \mathbb{R}^{d}:\|\mathbf{x}\|_{\mathrm{A}} \leq R\right\}$, i.e., (3.12) gives

$$
\|\mathbf{A} \mathbf{x}+\mathbf{d}\| \geq \rho\|\mathbf{x}\|-C \geq \frac{\rho^{2}+1}{\rho+1}\|\mathbf{x}\| .
$$

Condition (3.18) says that $\mathcal{S}^{(k)}$ stabilizes inside $\left\{\mathbf{x} \in \mathbb{R}^{d}:\|\mathbf{x}\|_{\mathrm{A}} \leq R\right\}$. By induction on $j \geq 0$ one obtains that $\mathcal{S}^{(k+j)}$ stabilizes inside the domain $\left\{\mathbf{x} \in \mathbb{R}^{d}:\|\mathbf{x}\|_{\mathrm{A}} \leq\right.$ $\left.\left(\left(\rho^{2}+1\right) /(\rho+1)\right)^{j} R\right\}$. The limit family $\mathcal{X}$ is discrete inside each of these domains, hence is discrete.

Lemma 3.6 leads to a recursively enumerable procedure which recognizes all irreducible discrete sets $\mathcal{X}$. More precisely, consider the class of inflation functional equations $\psi(\cdot)$ whose data $\left(\mathrm{A}, \mathcal{D}_{i j}\right)$ is drawn from a computable subfield $\mathbb{K}$ of $\mathbb{C}$, which is a field in which addition and multiplication are computable, and one can effectively test equality or inequality of field elements. Then all finite cycles $Y$ for such $\psi(\cdot)$ will have elements in $\mathbb{K}$. One can give a procedure which tests whether a given seed $\mathcal{S}^{(0)}$ generates an irreducible discrete $X$, using the criterion of Lemma 3.6. It will eventually halt for all such sets, and certify that they are discrete. (One does 
not need to compute $R$ or $\|\cdot\|_{\mathrm{A}}$ exactly to guarantee halting.) This procedure also detects all nonirreducible sets, by either finding a second cycle or a cycle with multiplicity. However, this procedure is not recursive, for given an input cycle $Y$ that generates an irreducible $\mathcal{X}$ which is not discrete, the procedure can run forever without halting.

The following lemma gives a case when all irreducible solutions to the inflation functional equation are discrete (if any exist).

Lemma 3.7. Suppose that the inflation functional equation $\psi(\cdot)$ has data $\left(\mathrm{A}, \mathcal{D}_{i j}\right)$, in which $\mathrm{A}$ is an expanding integer matrix and all vectors in $\mathcal{D}_{i j}$ have rational entries. Then all irreducible multiset families generated by a periodic cycle are discrete.

Proof. All cycles $Y$ of period $p$ are generated by solutions of the form (3.16), and the hypothesis guarantee that such a solution $\mathbf{x}$ is a rational vector. Starting from $(\mathbf{x}, i)$ one generates the orbit $Y$ and a seed $\mathcal{S}^{(0)}$ which consists of rational vectors. All elements of $\mathcal{S}^{(k)}$ are rational vectors with denominators dividing $d D$, where $d$ is the denominator of $\mathbf{x}$ and $D$ is the greatest common denominator of all the rationals in $\mathcal{D}_{i j}$. Thus there are only a finite number of possible choices for rational vectors in the ball of radius $R=R(\mathrm{~A}, \mathcal{D})$ in (3.11). Thus the criterion of Lemma 3.6 applies to conclude that if $\mathcal{X}=\lim _{k \rightarrow \infty} \mathcal{S}^{(k)}$ exists, then it is necessarily discrete.

Lemma 3.7 does not assert existence of irreducible multiset families, only that they are discrete if they do exist. Example 8.4 in Section 8 shows there are cases satisfying the hypotheses where no discrete solutions exist. At the other extreme, there are inflation functional equations of this type having infinitely many different irreducible discrete multiset families, see Example 8.3.

We conclude this section by raising two unsolved computational problems:

Computational Problem (i). Given a seed $\mathcal{S}_{0}$ which generates an irreducible discrete multiset family $\mathcal{X}$ satisfying a given inflation functional equation $\psi(\mathcal{X})=\mathcal{X}$ and an index $i$, determine whether the multiplicities of points in $X_{i}$ are bounded or not.

Computational Problem (ii). Given the same data as above, determine the maximum multiplicity of points in $X_{i}$.

\section{Perron Eigenvalue Condition}

In this section we consider inflation functional equations having a primitive subdivision matrix $S$ so that the Perron eigenvalue $\lambda(S)$ is defined. We show that a necessary condition for an inflation functional equation to have a solution that is a Delone set family is that the Perron eigenvalue $S$ satisfies the Perron eigenvalue condition

$$
\lambda(\mathrm{S})=|\operatorname{det}(\mathrm{A})|
$$


as given in Theorem 2.3. To achieve this we prove several preliminary results. Some of them apply more generally to multiset families $\mathcal{X}$ which are weakly uniformly discrete in the following sense.

Definition 4.1. A multiset family $\mathcal{X}$ is weakly uniformly discrete if the union $\bigvee_{i=1}^{n} X_{i}$ is a weakly uniformly discrete multiset.

A finite union of uniformly discrete multisets is weakly uniformly discrete if and only if each multiset itself is weakly uniformly discrete. So a multiset family $\mathcal{X}$ is weakly uniformly discrete if and only if each $X_{i}$ in it is weakly uniformly discrete.

We first consider an irreducible multiset family $\mathcal{X}$ satisfying $\mathcal{X}=\psi(\mathcal{X})$. Let $\mathcal{S}^{(0)}$ be the generating cycle for $\mathcal{X}$ and set $\mathcal{S}^{(k+1)}=\psi\left(\mathcal{S}^{(k)}\right)$. Write $\mathcal{S}^{(k)}=\left(S_{1}^{(k)}, S_{2}^{(k)}, \ldots, S_{n}^{(k)}\right)$ and let $H_{i}^{(k)}$ denote the total multiplicity of the multiset $\mathcal{S}_{i}^{(k)}$, which is given by

$$
H_{i}^{(k)}:=\sum_{\mathbf{x} \in \mathcal{S}^{(k)}} m_{S_{i}^{(k)}}(\mathbf{x}) .
$$

Lemma 4.1. Let $\mathcal{X}$ be an irreducible discrete multiset family satisfying $\psi(\mathcal{X})=\mathcal{X}$ for the data $\left(\mathrm{A}, \mathcal{D}_{i j}\right)$, having subdivision matrix $\mathrm{S}$. Let $\mathbf{H}^{(k)}=\left[H_{1}^{(k)}, \ldots, H_{n}^{(k)}\right]^{T}$. Then $\mathbf{H}^{(k)}=\mathrm{S}^{k} \mathbf{H}^{(0)}$.

Proof. We prove that $\mathbf{H}^{(k+1)}=\mathbf{S} \mathbf{H}^{(k)}$. Observe that

$$
S_{i}^{(k+1)}=\bigvee_{j=1}^{n}\left(S_{j}^{(k)}+\mathcal{D}_{i j}\right) .
$$

Hence

$$
H_{i}^{(k+1)}=\sum_{j=1}^{m}\left|\mathcal{D}_{i j}\right| H_{j}^{(k)}
$$

yielding $\mathbf{H}^{(k+1)}=\mathbf{S H}^{(k)}$. Therefore $\mathbf{H}^{(k)}=\mathrm{S}^{k} \mathbf{H}^{(0)}$.

Theorem 4.2. Let $\mathcal{X}$ be an irreducible discrete multiset family satisfying $\psi(\mathcal{X})=\mathcal{X}$ for the data $\left(\mathrm{A}, \mathcal{D}_{i j}\right)$, with a primitive subdivision matrix $\mathrm{S}=\left[\left|\mathcal{D}_{i j}\right|\right]$. Let $B_{1}(\mathbf{0})$ be the unit ball and set

$$
M_{i}^{(k)}:=\sum_{\mathbf{x} \in \underline{X}_{i} \cap A^{k}\left(B_{1}(\mathbf{0})\right)} m_{X_{i}}(\mathbf{x}) .
$$

Then there exist constants $C_{1}, C_{2}>0$ such that, for each $k \geq 1$,

$$
C_{1} \lambda(\mathrm{S})^{k} \leq M_{i}^{(k)} \leq C_{2} \lambda(\mathrm{S})^{k} .
$$

Proof. We first establish the lower bound. Observe that for each element $\mathbf{x} \in \bigvee_{i=1}^{n} S_{i}^{(k)}$ there exist digits $\mathbf{d}_{1}, \ldots, \mathbf{d}_{k}$ in the collection of digit sets $\left\{\mathcal{D}_{i j}\right\}$ such that 
$\mathbf{x}=\tau_{\mathbf{d}_{1}} \circ \cdots \circ \tau_{\mathbf{d}_{k}}\left(\mathbf{x}_{0}\right)$, where $\mathbf{x}_{0}$ is in the generating cycle and $\tau_{\mathbf{d}}(\mathbf{x}):=A \mathbf{x}+\mathbf{d}$. So

$$
\mathbf{x}=\mathrm{A}^{k} \mathbf{x}_{0}+\sum_{j=1}^{k} \mathrm{~A}^{j-1} \mathbf{d}_{j}=\mathrm{A}^{k}\left(\mathbf{x}_{0}+\sum_{j=1}^{k}\left(\mathrm{~A}^{-1}\right)^{k+1-j} \mathbf{d}_{j}\right) .
$$

Since $\mathrm{A}^{-1}$ is contracting, there exists a constant $C$ depending only on $\left\{\mathcal{D}_{i j}\right\}, A$ and $\mathcal{S}^{(0)}$ such that

$$
\left\|\mathbf{x}_{0}+\sum_{j=1}^{k}\left(\mathrm{~A}^{-1}\right)^{k+1-j} \mathbf{d}_{j}\right\| \leq C .
$$

Fix an integer $k_{1}>0$ so that $B_{C}(\mathbf{0}) \subseteq \mathrm{A}^{k_{1}}\left(B_{1}(\mathbf{0})\right)$. Then $\mathbf{x} \in \mathrm{A}^{k+k_{1}}\left(B_{1}(\mathbf{0})\right)$. It follows that for any $\mathbf{x} \in \underline{X}_{i} \cap \mathrm{A}^{k+k_{1}}\left(B_{1}(\mathbf{0})\right)$ we have

$$
m_{X_{i}}(\mathbf{x})=m_{S_{i}^{(k)}}(\mathbf{x}) .
$$

Therefore $M_{i}^{\left(k+k_{1}\right)} \geq H_{i}^{(k)}$ for all $k \geq 1$. By Lemma 4.1 and the primitivity of $S$ we have $H_{i}^{(k)} \geq C_{1}^{\prime} \lambda(\mathrm{S})^{k}$ for some constant $C_{1}^{\prime}$. The lower bound $M_{i}^{(k)} \geq C_{1} \lambda(\mathrm{S})^{k}$ follows by taking $C_{1}=C_{1}^{\prime} \lambda(\mathrm{S})^{-k_{1}}$.

To establish the upper bound we claim that there exists a $k_{2}$ such that for each $k \geq 1$, each $i$ and $\mathbf{x} \in \underline{X}_{i} \cap \mathrm{A}^{k}\left(B_{1}(\mathbf{0})\right)$ and any vertex $\mathbf{x} \in \underline{X}_{i}$ with $\mathbf{x} \in \mathrm{A}^{k}\left(B_{1}(\mathbf{0})\right)$ we must have

$$
m_{X_{i}}(\mathbf{x})=m_{S_{i}^{\left(k+k_{2}\right)}}(\mathbf{x}) .
$$

The claim gives the upper bound, for (4.4) implies that $M_{i}^{(k)} \leq H_{i}^{\left(k+k_{2}\right)}$ holds for each $k \geq 1$ and each $i$. However Lemma 4.1 and the primitivity of $S$ together show that there exists a constant $C_{2}^{\prime}$ such that

$$
M_{i}^{(k)} \leq C_{2}^{\prime} \lambda(\mathrm{S})^{k+k_{2}}
$$

Combining these inequalities gives the upper bound $M_{i}^{(k)} \leq C_{2} \lambda(\mathrm{S})^{k}$, on taking $C_{2}=$ $C_{2}^{\prime} \lambda(\mathrm{S})^{k_{2}}$.

It remains to prove (4.4). We note that any $\mathbf{x} \in \underline{X}_{i} \cap \mathrm{A}^{k}\left(B_{1}\right)$ must be in $S_{i}^{(l)}$ for some $l$ as a result of Lemma 3.1. Suppose that $l \geq k$. Then there exist some digits $\mathbf{d}_{1}, \mathbf{d}_{2}, \ldots, \mathbf{d}_{l}$ in $\mathcal{D}_{i j}$ 's such that

$$
\mathbf{x}=\mathrm{A}^{k} \mathbf{x}_{0}+\sum_{j=1}^{l} \mathrm{~A}^{j-1} \mathbf{d}_{j}
$$

and

$$
\mathbf{x}_{0}=\mathrm{A}^{-l} \mathbf{x}-\sum_{j=1}^{k} \mathrm{~A}^{-j} \mathbf{d}_{k+1-j} .
$$

Observe that $\mathbf{x}_{0}$ is bounded since $l \geq k, \mathrm{~A}^{-k} \mathbf{x} \in B_{1}$, and $\mathrm{A}$ is expanding. Hence there exists a constant $R_{0}$ independent of $k$ and $l$ such that $\left\|\mathbf{x}_{0}\right\| \leq R_{0}$. Let

$$
k_{2}=\sum_{i=1}^{n}\left|\underline{X}_{i} \cap B_{R_{0}}(\mathbf{0})\right|
$$


We now argue by contradiction, and assume that (4.4) is false. Then there exist some $k \geq 1, i$ and $\mathbf{x} \in \underline{X}_{i} \cap \mathrm{A}^{k}\left(B_{1}\right)$ such that $m_{X_{i}}(\mathbf{x})>m_{S_{i}^{\left(k+k_{2}\right)}}(\mathbf{x})$. This means there exists a cycle-free directed path in $\mathcal{G}(\mathcal{X})$ of length $l>k+k_{2}$ from an element $\mathbf{x}_{0}$ in the generating cycle $\mathcal{S}^{(0)}$ to $\mathbf{x}$, say $\mathbf{x}=\tau_{\mathbf{d}_{l}} \circ \cdots \circ \tau_{\mathbf{d}_{2}} \circ \tau_{\mathbf{d}_{1}}\left(\mathbf{x}_{0}\right)$. Let $\mathbf{x}_{j}=\tau_{\mathbf{d}_{j}}\left(\mathbf{x}_{j-1}\right), 1 \leq j \leq l$, be the vertices of this directed path. It follows that $\left\|\mathbf{x}_{j}\right\| \leq R_{0}$ for $0 \leq j \leq l-k$. However, there are only $k_{2}$ vertices in $B_{R_{0}}(\mathbf{0})$ and $l-k>k_{2}$, hence there exist two identical vertices among $\left\{\mathbf{x}_{j}: 1 \leq j \leq l-k\right\}$. This contradicts the assumption that the directed path is cycle-free, proving (4.4).

Theorem 4.3. Let $\mathcal{X}$ be a weakly uniformly discrete $n$-multiset family satisfying $\psi(\mathcal{X})=\mathcal{X}$ for the data $\left(\mathrm{A}, \mathcal{D}_{i j}\right)$, with a primitive subdivision matrix $\mathrm{S}$. Then

$$
\lambda(\mathrm{S}) \leq|\operatorname{det}(\mathrm{A})|
$$

Proof. We first assume that $\mathcal{X}$ is irreducible. The weak uniform discreteness of $\mathcal{X}$ implies that $M_{i}^{(k)} \leq C \operatorname{Vol}\left(\mathrm{A}^{k} B_{1}(\mathbf{0})\right)=C|\operatorname{det}(\mathrm{A})|^{\mathrm{k}}$ for some positive constant $C$. From Theorem 4.2 it immediately follows that $|\operatorname{det}(\mathrm{A})| \geq \lambda(\mathrm{S})$.

If $\mathcal{X}$ is reducible, then $\mathcal{X}=\bigvee_{j=1}^{N} \mathcal{X}^{(j)}$. The argument above now applies to $\mathcal{X}^{(1)}$ to yield $\lambda(\mathrm{S}) \leq|\operatorname{det}(\mathrm{A})|$.

Proof of Theorem 2.3. Since $\mathcal{X}$ is a weak substitution Delone multiset family, it is weakly uniformly discrete. By Theorem 4.3 this immediately gives $\lambda(\mathrm{S}) \leq|\operatorname{det}(\mathrm{A})|$.

To prove the other direction, $\lambda(\mathrm{S}) \geq|\operatorname{det}(\mathrm{A})|$, let $\mathcal{X}=\bigvee_{j=1}^{N} \mathcal{X}^{(j)}$. Let $M_{i, j}^{(k)}$ be as in (4.2), but defined for $\mathcal{X}^{(j)}$. Then the relative denseness of each $X_{i}$ yields

$$
\sum_{j=1}^{N} M_{i, j}^{(k)} \geq C^{\prime} \operatorname{Vol}\left(\mathrm{A}^{k} B_{1}(\mathbf{0})\right)=C^{\prime}|\operatorname{det}(\mathrm{A})|^{\mathrm{k}}
$$

for some positive constant $C^{\prime}$. Hence $\max _{j} M_{i, j}^{(k)} \geq(1 / N) C^{\prime}|\operatorname{det}(\mathrm{A})|^{\mathrm{k}}$. Taking the $k$ th roots and letting $k \rightarrow \infty$ now yields $\lambda(\mathrm{S}) \geq|\operatorname{det}(\mathrm{A})|$, using Theorem 4.2.

\section{Multi-Tiling Functional Equation}

Our object in the next section will be to relate the existence of weak substitution Delone multiset families to self-replicating tilings. In this section we obtain preliminary information, concerning solutions of the multi-tiling functional equation. Theorem 5.2 gives a necessary condition for solutions of positive Lebesgue measure and Theorem 5.5 gives a set of equivalent conditions for positive Lebsegue measure that apply when the Perron eigenvalue condition $\lambda(\mathrm{S})=|\operatorname{det}(\mathrm{A})|$ holds.

We begin with a basic existence result on solutions to the multi-tiling equation.

Proposition 5.1. The multi-tile functional equation

$$
\mathrm{A}\left(T_{i}\right)=\bigcup_{j=1}^{n}\left(T_{j}+\mathcal{D}_{j i}\right), \quad 1 \leq i \leq n,
$$


with a primitive subdivision matrix $\mathrm{S}$ has a unique nonempty solution $\left(T_{1}, \ldots, T_{n}\right)$ in which each $T_{i}$ is compact. In this solution all $T_{i}$ are nonempty, and

$$
T_{i}=\left\{\sum_{k=1}^{\infty} \mathrm{A}^{-k} \mathbf{d}_{j_{k} j_{k-1}} \mid \mathbf{d}_{j_{k} j_{k-1}} \in \mathcal{D}_{j_{k} j_{k-1}},\left(j_{0}, j_{1}, j_{2}, \ldots\right) \in\{1,2, \ldots, N\}^{\mathbb{Z}^{+}}, j_{0}=i\right\} .
$$

Proof. Flaherty and Wang [1, Proposition 2.3] prove under the hypothesis

$$
\bigcup_{j=1}^{n} \mathcal{D}_{j i} \quad \text { is nonempty for } \quad 1 \leq i \leq n,
$$

that the multi-tile equation (5.1) has a unique nonempty solution $\left(T_{1}, \ldots, T_{n}\right)$ in which all $T_{i}$ are compact sets, and that in this solution all $T_{i}$ are nonempty. The primitivity assumption on $\mathrm{S}$ implies that hypothesis $(*)$ holds, hence this result applies.

We remark that (5.2) has a graph-theoretic interpretation. Form a directed graph $\mathcal{G}(\psi)$ whose vertices correspond to sets $T_{i}$ and with a directed edge from $T_{i}$ to $T_{j}$ labeled $\mathbf{d}$ if $\mathbf{d} \in \mathcal{D}_{j i}$. Then (5.2) is equivalent to saying that for each point $\mathbf{x} \in T_{i}$ there exists an infinite directed path $\left(\mathbf{d}_{1}, \mathbf{d}_{2}, \mathbf{d}_{3}, \ldots\right)$ in the graph $\mathcal{G}(\psi)$ where an edge connects with $\mathbf{d}_{1} \in \mathcal{D}_{j i}$ for some $j$ such that

$$
\mathbf{x}=\sum_{k=1}^{\infty} \mathrm{A}^{-k} \mathbf{d}_{k}
$$

and vice versa.

We next give a simple necessary condition for the sets $T_{i}$ to have positive Lebesgue measure.

Theorem 5.2. Suppose that the multi-tile functional equation

$$
\mathrm{A}\left(T_{i}\right)=\bigcup_{j=1}^{n}\left(T_{j}+\mathcal{D}_{j i}\right), \quad 1 \leq i \leq n,
$$

with a primitive subdivision matrix $\mathrm{S}=\left[\left|\mathcal{D}_{i j}\right|\right]$ has a nonempty solution $\left(T_{1}, \ldots, T_{n}\right)$ consisting of compact sets, in which at least one $T_{i}$ has positive Lebesgue measure. Then

$$
\lambda(\mathrm{S}) \geq|\operatorname{det}(\mathrm{A})| \text {. }
$$

Proof. Taking the Lebesgue measure of both sides of the multi-tile equation (5.4) gives, for $1 \leq i \leq n$,

$$
|\operatorname{det}(\mathrm{A})| \mu\left(T_{i}\right) \leq \sum_{j=1}^{n}\left|\mathcal{D}_{j i}\right| \mu\left(T_{j}\right),
$$

where $\left|\mathcal{D}_{j i}\right|$ denotes the cardinality of $\mathcal{D}_{j i}$ (counting multiplicity). By hypothesis $\mathbf{v}:=\left[\mu\left(T_{1}\right), \ldots, \mu\left(T_{n}\right)\right]$ is a nonnegative row vector, not identially zero, and the equation above gives

$$
\mathbf{v S} \geq|\operatorname{det}(\mathrm{A})| \mathbf{v} .
$$


Write $\mathbf{v} S=|\operatorname{det}(A)| \mathbf{v}+\mathbf{w}_{1}$, where $\mathbf{w}_{1}$ is nonnegative. Repeated multiplication by $\mathbf{S}$ and back-substitution yields $\mathbf{v} S^{k}=|\operatorname{det}(\mathrm{A})|^{\mathrm{k}} \mathbf{v}+\mathbf{w}_{\mathrm{k}}$, where $\mathbf{w}_{k}$ is nonnegative. We conclude that the spectral radius $\rho(\mathrm{S})$ satisfies

$$
\rho(\mathrm{S}) \geq \lim _{k \rightarrow \infty}\left\|\mathbf{v} S^{k}\right\|^{1 / k} \geq|\operatorname{det}(\mathrm{A})| .
$$

Thus $\lambda(\mathrm{S}) \geq|\operatorname{det}(\mathrm{A})|$.

In the remainder of this section we develop criteria for positive Lebesgue measures of the sets $T_{i}$ that apply when the Perron eigenvalue condition $\lambda(\mathrm{S})=|\operatorname{det}(\mathrm{A})|$ holds.

We define the digit multisets

$$
\mathcal{D}_{j i}^{m}:=\bigvee_{j_{1}, \ldots, j_{m-1}=1}^{n}\left(\mathcal{D}_{j j_{m-1}}+\mathrm{AD}_{j_{m-1} j_{m-2}}+\cdots+\mathrm{A}^{m-1} \mathcal{D}_{j_{1} i}\right),
$$

in which the sum is interpreted as counting multiplicities. It is easy to check that iterating (5.1) yields

$$
\mathrm{A}^{m}\left(T_{i}\right)=\bigcup_{j=1}^{n}\left(T_{j}+\underline{\mathcal{D}}_{j i}^{m}\right), \quad i=1, \ldots, n .
$$

In (5.7) we do not count multiplicity, so it suffices to use $\underline{\mathcal{D}}_{j i}^{m}$ instead of $\mathcal{D}_{j i}^{m}$.

Definition 5.1. (i) A family of discrete multisets $\left\{\mathcal{E}_{\alpha}: \alpha \in I\right\}$ in $\mathbb{R}^{d}$ is equi-uniformly discrete if there exists $\varepsilon_{0}>0$ such that each $\mathcal{E}_{\alpha}$ is uniformly discrete and any two distinct elements in $\mathcal{E}_{\alpha}$ are separated by at least distance $\varepsilon_{0}$. In particular, each $\mathcal{E}_{\alpha}$ is an ordinary set.

(ii) A family of discrete multisets $\left\{\mathcal{E}_{\alpha}: \alpha \in I\right\}$ is called weakly equi-uniformly discrete if there exists a fixed $M>0$ such that for each $\alpha \in I$ and each ball $B$ of radius 1 in $\mathbb{R}^{d}$ the number of elements of $\mathcal{E}_{\alpha}$ in $B$ (counting multiplicity) is bounded by $M$.

The following theorem is an extension of a theorem in [34]. Its hypotheses can only be satisfied when the Perron eigenvalue condition holds, because the weak equi-uniformly discrete hypothesis can only hold when $\lambda(S) \leq|\operatorname{det}(A)|$.

Theorem 5.3. Suppose that the compact sets $\left(T_{1}, \ldots, T_{n}\right)$ satisfy the multi-tile functional equation

$$
\mathrm{A}\left(T_{i}\right)=\bigcup_{j=1}^{n}\left(T_{j}+\mathcal{D}_{j i}\right), \quad i=1, \ldots, n,
$$

with a primitive subdivision matrix $\mathrm{S}$, and that at least one $T_{i}$ has positive Lebesgue measure. If the collection of multisets $\left\{\underline{\mathcal{D}}_{j i}^{m}: 1 \leq i, j \leq n, m \geq 1\right\}$ are weakly equiuniformly discrete, then each $T_{i}$ has nonempty interior $T_{i}^{\circ}$, and is the closure of its interior, $T_{i}=\overline{T_{i}^{\circ}}$.

The primitivity of the subdivision matrix implies that if a single $T_{i}$ has positive Lebesgue measure, then they all do. The proof of Theorem 5.3 is based on the following covering lemma. 
Lemma 5.4. Suppose that $\left(T_{1}, \ldots, T_{n}\right)$ are compact sets satisfying an inflation functional equation with a primitive subdvision matrix, and that at least one $T_{i}$ has positive Lebesgue measure. Suppose also that the collection of multisets $\left\{\mathcal{D}_{j i}^{m}: 1 \leq i, j \leq n\right.$, $m \geq 1\}$ is weakly equi-uniformly discrete. Then given any sequence of positive numbers $\left\{\delta_{m}\right\}$ with $\lim _{m \rightarrow \infty} \delta_{m}=0$, there exist positive constants $R_{0}$ and $K_{0}$ such that the following holds: For each $m \geq 1$ there exist subsets $\mathcal{E}_{1}^{m}, \ldots, \mathcal{E}_{n}^{m}$ of $\mathbb{R}^{d}$ contained in the ball $B_{R_{0}}(\mathbf{0})$, each of cardinality bounded by $K_{0}$, such that $\Omega_{m}:=\bigcup_{j=1}^{n}\left(T_{j}+\mathcal{E}_{j}^{m}\right)$ has the property that

$$
\mu\left(B_{1}(\mathbf{0}) \cap \Omega_{m}\right) \geq\left(1-5^{d+1} \delta_{m}\right) \mu\left(B_{1}(\mathbf{0})\right) .
$$

Proof. Without loss of generality we assume $T_{1}$ has positive Lebesgue measure, so $T_{1}$ has a Lebesgue point $\mathbf{x}^{*}$, i.e., there is a sequence $r_{m} \rightarrow 0$ such that

$$
\mu\left(B_{r_{m}}\left(\mathbf{x}^{*}\right) \cap T_{1}\right) \geq\left(1-\delta_{m}\right) \mu\left(B_{r_{m}}\left(\mathbf{x}^{*}\right)\right) .
$$

It follows that

$$
\mu\left(\mathrm{A}^{l}\left(B_{r_{m}}\left(\mathbf{x}^{*}\right) \cap T_{1}\right)\right) \geq\left(1-\delta_{m}\right) \mu\left(\mathrm{A}^{l}\left(B_{r_{m}}\left(\mathbf{x}^{*}\right)\right)\right), \quad \text { for all } \quad l \geq 0 .
$$

We first show that for sufficiently large $l=l(m)$, there exists a unit ball $B_{1}(\mathbf{y}) \subset$ $\mathrm{A}^{l}\left(B_{r_{m}}\left(\mathbf{x}^{*}\right)\right)$ with

$$
\mu\left(B_{1}(\mathbf{y}) \cap \mathrm{A}^{l}\left(T_{1}\right)\right) \geq\left(1-5^{d+1} \delta_{m}\right) \mu\left(B_{1}(\mathbf{0})\right) .
$$

Indeed, since $\mathrm{A}$ is expanding, $\mathrm{A}^{l}\left(B_{r_{m}}\left(\mathbf{x}^{*}\right)\right)$ is an ellipsoid $O_{l, m}$ whose shortest axis goes to infinity as $l$ goes to infinity. Let $O_{l, m}^{\prime}$ be the homothetically shrunk ellipsoid with shortest axis decreased in length by 2 , so that all points in it are at distance at least 1 from the boundary of $O_{l, m}$. By a standard covering lemma (see p. 9 of [38]) applied to $O_{l, m}^{\prime}$ there is a set $\left\{B_{1}\left(\mathbf{y}^{\prime}\right)\right\}$ of disjoint unit balls with centers in $O_{l, m}^{\prime}$ that cover volume at least $5^{-d} \mu\left(O_{l, m}^{\prime}\right)$. Also $5^{-d} \mu\left(O_{l, m}^{\prime}\right) \geq 5^{-d-1} \mu\left(O_{l, m}\right)$ once the shortest axis is of length at least $2(d+1)$. All these balls lie inside $O_{l, m}$. By (5.9) at most $\delta_{m} \mu\left(\mathrm{A}^{l}\left(B_{r_{m}}\left(\mathbf{x}^{*}\right)\right)\right)$ of the volume of $\mathrm{A}^{l}\left(B_{r_{m}}\left(\mathbf{x}^{*}\right)\right)$ is uncovered by $\mathrm{A}^{l}\left(B_{r_{m}}\left(\mathbf{x}^{*}\right) \cap T_{1}\right)$, so at least one of the disjoint balls $\left\{B_{1}\left(\mathbf{y}^{\prime}\right)\right\}$ must satisfy (5.10).

By (5.6) we can rewrite inequality (5.10) as

$$
\mu\left(B_{1}(\mathbf{y}) \cap\left(\bigcup_{j=1}^{n}\left(T_{j}+\underline{\mathcal{D}}_{j 1}^{l}\right)\right)\right) \geq\left(1-5^{d+1} \delta_{m}\right) \mu\left(B_{1}(\mathbf{0})\right),
$$

whence

$$
\mu\left(B_{1}(\mathbf{0}) \cap\left(\bigcup_{j=1}\left(T_{j}+\underline{\mathcal{D}}_{j 1}^{l}-\mathbf{y}\right)\right)\right) \geq\left(1-5^{d+1} \delta_{m}\right) \mu\left(B_{1}(\mathbf{0})\right) .
$$

This shows that if we choose

$$
\mathcal{E}_{j}^{m}:=\left\{\mathbf{d}-\mathbf{y} \mid \mathbf{d} \in \underline{\mathcal{D}}_{j 1}^{l} \text { with }\left(T_{j}+\mathbf{d}-\mathbf{y}\right) \cap B_{1}(\mathbf{0}) \neq \emptyset\right\},
$$

then (5.8) holds. Since all $T_{j}$ are compact, all $\mathcal{E}_{j}^{m}$ lie inside the ball $B_{R_{0}}(\mathbf{0})$ with $R_{0}:=$ $1+\max _{i} \operatorname{diam}\left(T_{i}\right)$. The cardinality of all $\mathcal{E}_{j}^{m}$ are upper bounded by a constant $K_{0}$ because the set of all $\underline{\mathcal{D}}_{i j}^{m}$ is weakly equi-uniformly discrete. 
Proof of Theorem 5.3. We apply Lemma 5.4 and choose a subsequence $m_{k} \rightarrow \infty$ so that $\left\{\mathcal{E}_{j}^{m_{k}}\right\}$ converges (as a multiset) for all $j$, and we denote the limit multisets by $\mathcal{E}_{j}^{\infty}$. This can always be done because $\left\{\mathcal{E}_{j}^{m}\right\}$ are uniformly bounded and have uniformly bounded cardinality. Clearly, $\mathcal{E}_{j}^{\infty}$ has cardinality at most $K_{0}$. So

$$
\begin{aligned}
\mu\left(B_{1}(\mathbf{0}) \cap\left(\bigcup_{j=1}^{n}\left(T_{j}+\mathcal{E}_{j}^{\infty}\right)\right)\right) & \geq \liminf _{k \rightarrow \infty} \mu\left(B_{1}(\mathbf{0}) \cap\left(\bigcup_{j=1}^{n}\left(T_{j}+\mathcal{E}_{j}^{m_{k}}\right)\right)\right) \\
& \geq \liminf _{k \rightarrow \infty}\left(1-5^{d+1} \delta_{m_{k}}\right) \mu\left(B_{1}(\mathbf{0})\right) \\
& =\mu\left(B_{1}(\mathbf{0})\right) .
\end{aligned}
$$

Since each $T_{j}+\mathcal{E}_{j}^{\infty}$ is a closed set, we must have

$$
B_{1}(\mathbf{0}) \cap\left(\bigcup_{j=1}^{n}\left(T_{j}+\mathcal{E}_{j}^{\infty}\right)\right)=B_{1}(\mathbf{0}) .
$$

This means that at least one of the $T_{j}$ 's must have nonempty interior $T_{j}^{\circ}$. However, if so, then the primitivity of the subdivision matrix implies that all $T_{j}$ have nonempty interior. Let $T_{j}^{\prime}=\overline{T_{j}^{\circ}}$. Then $\left(T_{1}^{\prime}, \ldots, T_{n}^{\prime}\right)$ must also satisfy the same multi-tile equation (5.1). By the uniqueness assertion in Proposition 5.1 we have $T_{j}=T_{j}^{\prime}$ for $1 \leq j \leq n$.

We obtain the following characterization of the positive measure property $\mu\left(T_{i}\right)>0$, which applies when the Perron eigenvalue condition holds.

Theorem 5.5. Assume that the family of compact sets $\left(T_{1}, \ldots, T_{n}\right)$ satisfies a multi-tile functional equation with the data $\left(\mathrm{A}, \mathcal{D}_{i j}\right)$ with primitive subdivision matrix $\mathrm{S}$ satisfying the Perron eigenvalue condition $\lambda(\mathrm{S})=|\operatorname{det}(\mathrm{A})|$. Then the following conditions are equivalent:

(i) For $m \geq 1$ all multisets $\mathcal{D}_{j k}^{m}$ for $1 \leq i, j \leq n$ are ordinary sets, and the family $\left\{\mathcal{D}_{j k}^{m}: 1 \leq i, j \leq n, m \geq 1\right\}$ is equi-uniformly discrete.

(ii) For some fixed $1 \leq k, l \leq n$, and $m \geq 1$ the multisets $\mathcal{D}_{k l}^{m}$ are ordinary sets and the family $\left\{\mathcal{D}_{k l}^{m}: m \geq 1\right\}$ is equi-uniformly discrete.

(iii) The family of multisets $\left\{\mathcal{D}_{j k}^{m}: 1 \leq i, j \leq n, m \geq 1\right\}$ is weakly equi-uniformly discrete.

(iv) For some fixed $k, l(1 \leq k, l \leq n)$ the family of multisets $\left\{\mathcal{D}_{k l}^{m}: m \geq 1\right\}$ is weakly equi-uniformly discrete.

(v) One set $T_{j}$ has $\mu\left(T_{j}\right)>0$.

(vi) Every set $T_{j}$ has $T_{j}=\overline{T_{j}^{\circ}}$, hence all $\mu\left(T_{j}\right)>0$.

(vii) Each $T_{j}=\overline{T_{j}^{\circ}}$, for $1 \leq j \leq n$, and $\mu\left(\partial T_{k}\right)=0$.

Proof. The implications (i) $\Rightarrow$ (ii) $\Rightarrow$ (iv) and (i) $\Rightarrow$ (iii) $\Rightarrow$ (iv) are obvious.

(iv) $\Rightarrow$ (v) First it is easy to check that $S^{m}=\left[\left|\mathcal{D}_{i j}^{m}\right|\right]$ where $\left|\mathcal{D}_{i j}^{m}\right|$ denotes the cardinality (counting multiplicity) of $\mathcal{D}_{i j}^{m}$. The primitivity hypothesis implies that there exists a 
$c_{0}>0$ such that, for all $m \geq 1,\left|\mathcal{D}_{i j}^{m}\right| \geq c_{0} \lambda(\mathrm{S})^{m}$ for all $1 \leq i, j \leq n$. Now for all $i$ let $T_{i}^{(0)}=\overline{B_{1}(\mathbf{0})}$ and

$$
T_{i}^{(m)}=\bigcup_{j=1}^{n} \mathrm{~A}^{-1}\left(T_{j}^{(m-1)}+\mathcal{D}_{j i}\right), \quad m>0 .
$$

Then $T_{i}^{(m)} \longrightarrow T_{i}$ in the Hausdorff metric (see [1]). We prove that $\mu\left(T_{l}\right)>0$ for all $1 \leq l \leq n$. To see this, we note that

$$
\mathrm{A}^{m}\left(T_{i}^{(m)}\right)=\bigcup_{j=1}^{n}\left(T_{j}^{(0)}+\underline{\mathcal{D}}_{j i}^{m}\right) .
$$

Then

$$
\mathrm{A}^{m}\left(T_{l}^{(m)}\right)=\bigcup_{j=1}^{n}\left(T_{j}^{(0)}+\underline{\mathcal{D}}_{j l}^{m}\right) \supseteq T_{k}^{(0)}+\underline{\mathcal{D}}_{k l}^{m} .
$$

Since $\left\{\mathcal{D}_{k l}^{m}\right\}$ are weakly equi-uniformly discrete, there exists a constant $c_{1}>0$ such that $\left|\underline{\mathcal{D}}_{k l}^{m}\right| \geq c_{1}\left|\mathcal{D}_{k l}^{m}\right|$ for all $m$. Now since $\underline{\mathcal{D}}_{k l}^{m}$ are weakly equi-uniformly discrete there exists an $M>0$ such that each unit ball contains at most $M$ elements of $\mathcal{D}_{k l}^{m}$. Therefore each point in $T_{k}^{(0)}+\underline{\mathcal{D}}_{k l}^{m}$ can be covered by no more than $M$ copies of $T_{k}^{(0)}+\mathbf{d}, \mathbf{d} \in \underline{\mathcal{D}}_{k l}^{m}$. Then by $(5.11)$

$$
|\operatorname{det}(\mathrm{A})|^{m} \mu\left(T_{l}^{(m)}\right) \geq \frac{1}{M}\left|\underline{\mathcal{D}}_{k l}^{m}\right| \mu\left(T_{l}^{(0)}\right) \geq \frac{1}{M} \delta c_{0} c_{1} \lambda(\mathrm{S})^{m},
$$

where $\delta$ is the volume of the ball $B_{1}(\mathbf{0})$. Using the hypothesis $\lambda(\mathrm{S})=|\operatorname{det}(\mathrm{A})|$ yields

$$
\mu\left(T_{l}^{(m)}\right) \geq \frac{1}{M} \delta c_{0} c_{1}>0
$$

It follows that, for all $l$,

$$
\mu\left(T_{l}\right) \geq \liminf _{m \rightarrow \infty} \mu\left(T_{l}^{(m)}\right)>0 .
$$

(v) $\Rightarrow$ (i) First we note that $\mu\left(T_{j}\right)>0$ holds for all $j$ using the primitivity of $\mathrm{S}$. Let $\mathbf{e}=\left[\mu\left(T_{1}\right), \mu\left(T_{2}\right), \ldots, \mu\left(T_{n}\right)\right]$. Taking the Lebesgue measure of both sides of the iterated multi-tile equation (5.7) yields

$$
\lambda^{m}(\mathrm{~S}) \mu\left(T_{i}\right) \leq \sum_{j=1}^{n} \mu\left(T_{j}\right)\left|\underline{\mathcal{D}}_{j i}^{m}\right| \leq \sum_{j=1}^{n} \mu\left(T_{j}\right)\left|\mathcal{D}_{j i}^{m}\right|, \quad 1 \leq i \leq n .
$$

In other words, $\lambda^{m}(\mathbf{S}) \mathbf{e} \leq \mathbf{e} \mathbf{S}^{m}$. However, $\mathbf{S}^{m}$ is a primitive nonnegative matrix with Perron-Frobenius eigenvalue $\lambda^{m}(S)$. So (5.12) can hold only when e is a left PerronFrobenius eigenvector of $S$ and all inequalities in (5.12) are equalities. This immediately yields $\left|\mathcal{D}_{j i}^{m}\right|=\left|\underline{\mathcal{D}}_{j i}^{m}\right|$, hence each $\mathcal{D}_{j i}^{m}$ is an ordinary set. The equalities in (5.12) also imply that the unions $T_{j}+\underline{\mathcal{D}}_{j i}^{m}$ are all measurewise disjoint. So all $\underline{D}_{j i}^{m}$, and hence all $\mathcal{D}_{j i}^{m}$, are equi-uniformly discrete for some $\varepsilon>0$. 
(v) $\Leftrightarrow$ (vi) This follows from Theorem 5.3, since (v) also implies (i).

(vi) $\Rightarrow$ (vii) We only need to prove that $\mu\left(\partial T_{j}\right)=0$ for all $j$. Let $\mathbf{v}=\left[\mu\left(\partial T_{1}\right), \ldots\right.$, $\left.\mu\left(\partial T_{n}\right)\right]$. We have, for all $i$,

$$
\mathrm{A}^{m}\left(\partial T_{i}\right)=\partial\left(\mathrm{A}^{m}\left(T_{i}\right)\right)=\partial\left(\bigcup_{j=1}^{n}\left(T_{j}+\underline{\mathcal{D}}_{j i}^{m}\right)\right) \subseteq \bigcup_{j=1}^{n}\left(\partial T_{j}+\underline{\mathcal{D}}_{j i}^{m}\right)
$$

Taking the Lebesgue measure yields $\lambda^{m}(\mathrm{~S}) \mathbf{v} \leq \mathbf{v} \mathbf{S}^{m}$, similarly to (5.12). Again, this can occur only when $\mathbf{v}=\mathbf{0}$ or $\mathbf{v}$ is a Perron-Frobenius left eigenvector of $\mathbf{S}^{m}$. Assume that $\mathbf{v} \neq \mathbf{0}$ then all $\mu\left(\partial T_{j}\right)>0$, and

$$
\mu\left(\partial\left(\mathrm{A}^{m}\left(T_{i}\right)\right)\right)=\sum_{j=1}^{n}\left|\mathcal{D}_{j i}^{m}\right| \mu\left(\partial T_{j}\right)
$$

However, $T_{i}$ has a nonempty interior, so for sufficiently large $m>0$ the inflated set $\mathrm{A}^{m}\left(T_{i}\right)$ will contain a large ball in its interior, at least twice the diameter of any $T_{k}$. Since $\mathrm{A}^{m}\left(T_{i}\right)$ is the union of $T_{j}+\underline{\mathcal{D}}_{j i}^{m}, 1 \leq j \leq n$, there must be some $k$ and $\mathbf{d} \in \underline{\mathcal{D}}_{k i}$ such that $T_{k}+\mathbf{d}$ is completely contained in the interior of $\mathrm{A}^{m}\left(T_{i}\right)$. Hence

$$
\partial\left(\mathrm{A}^{m}\left(T_{i}\right)\right) \subseteq \bigcup_{j=1}^{n}\left(\partial T_{j}+\underline{\mathcal{D}}_{j i}^{m}\right) \backslash\left(T_{k}+\mathbf{d}\right)
$$

So (5.13) is impossible, a contradiction.

(vii) $\Rightarrow$ (vi) This is obvious.

\section{Weakly Uniformly Discrete Multiset Families and Tilings}

In this section we suppose that the Perron eigenvalue condition $\lambda(S)=|\operatorname{det}(A)|$ holds. Using the results of Section 5 we relate the existence of positive Lebesgue measure tiles $T_{i}$ for the associated multi-tiling equation to weakly uniformly discrete multiset solutions and self-replicating multi-tiling solutions to some iterate $\psi^{N}(\cdot)$ of the inflation functional equation. Then we deduce Theorem 2.4.

Theorem 6.1. Let $\psi(\mathcal{X})=\mathcal{X}$ be an inflation functional equation that has a primitive subdivision matrix $\mathrm{S}$ that satisfies the Perron eigenvalue condition

$$
\lambda(\mathrm{S})=|\operatorname{det}(\mathrm{A})|
$$

Then the following conditions are equivalent:

(i) For some $N>0$ there exists a weakly uniformly discrete multiset family $\hat{\mathcal{X}}$ such that $\psi^{N}(\hat{\mathcal{X}})=\hat{\mathcal{X}}$.

(ii) For some $N>0$ there exists a uniformly discrete multiset family $\hat{\mathcal{X}}$ such that $\psi^{N}(\hat{\mathcal{X}})=\hat{\mathcal{X}}$

(iii) For some $N>0$ there exists a self-replicating multi-tiling family $\hat{\mathcal{X}}$ with $\psi^{N}(\hat{\mathcal{X}})=\hat{\mathcal{X}}$. 
(iv) The unique compact solution $\left(T_{1}, \ldots, T_{n}\right)$ of the associated multi-tile functional equation consists of sets $T_{i}$ that all have positive Lebesgue measure.

Proof. Iterating $N$ times the inflation functional equation $\mathcal{X}=\psi(\mathcal{X})$ on multisets gives a new inflation functional equation $\mathcal{X}=\psi^{N}(\mathcal{X})$, which corresponds to

$$
X_{i}=\bigvee_{j=1}^{n}\left(\mathrm{~A}^{N}\left(X_{j}\right)+\mathcal{D}_{i j}^{N}\right), \quad 1 \leq i \leq n
$$

where the sums are interpreted as multiset sums.

We show that (i) $\Rightarrow$ (iv) $\Rightarrow$ (iii) $\Rightarrow$ (ii) $\Rightarrow$ (i). To begin, the implications (iii) $\Rightarrow$ (ii) $\Rightarrow$ (i) are obvious.

(i) $\Rightarrow$ (iv) By assumption there exists an $M>0$ such that any unit ball in $\mathbb{R}^{d}$ contains at most $M$ elements (counting multiplicity) of each $X_{i}$. Replacing $N$ by $m N$ in (6.1) it follows that any unit ball contains at most $M$ elements of each $\mathcal{D}_{i j}^{m N}$. Observe that by (5.6) we have

$$
\mathcal{D}_{i j}^{k}=\bigvee_{l=1}^{n}\left(\mathcal{D}_{i l}^{k-1}+\mathrm{A}^{k-1} \mathcal{D}_{l j}\right)
$$

Therefore any unit ball contains at most $M$ elements of each $\mathcal{D}_{i j}^{k-1}$ if it contains at most $M$ elements of each $\mathcal{D}_{i j}^{k}$. This immediately yields the weakly equi-uniform discreteness of all the sets $\left\{\mathcal{D}_{i j}^{k}\right\}$. So $T_{i}^{\circ} \neq \emptyset$ by Theorem 5.5.

(iv) $\Rightarrow$ (iii) Since $T_{1}^{\circ} \neq \varnothing$ it follows from the observation (5.3) that there exists an infinite directed path $\left(\mathbf{d}_{1}, \mathbf{d}_{2}, \mathbf{d}_{3}, \ldots\right)$ in the graph $\mathcal{G}(\mathcal{X})$ with $\mathbf{d}_{1} \in \mathcal{D}_{j 1}$ for some $j$ such that

$$
\mathbf{x}_{0}=\sum_{k=1}^{\infty} \mathrm{A}^{-k} \mathbf{d}_{k} \in T_{1}^{\circ}
$$

Since all $\mathcal{D}_{i j}$ are bounded, there exists an $N^{\prime}>0$ such that for all infinite directed paths $\left(\mathbf{d}_{1}^{\prime}, \mathbf{d}_{2}^{\prime}, \mathbf{d}_{3}^{\prime}, \ldots\right)$ with $\mathbf{d}_{j}^{\prime}=\mathbf{d}_{j}$ for $j \leq N^{\prime}$ we also have

$$
\mathbf{x}_{0}^{\prime}=\sum_{k=1}^{\infty} \mathrm{A}^{-k} \mathbf{d}_{k}^{\prime} \in T_{1}^{\circ}
$$

The primitivity of the subdivision matrix $S$ now implies that we can find an infinite directed path $\left(\mathbf{d}_{1}^{*}, \mathbf{d}_{2}^{*}, \mathbf{d}_{3}^{*}, \ldots\right)$ which has $\mathbf{d}_{j}^{*}=\mathbf{d}_{j}$ for $j \leq N^{\prime}$, and which is periodic for some period $N \geq N^{\prime}$ in the sense that $\mathbf{d}_{k+N}^{*}=\mathbf{d}_{k}^{*}$ for all $k$. Let $\mathbf{x}_{0}^{*}=\sum_{k=1}^{\infty} \mathrm{A}^{-k} \mathbf{d}_{k}^{*}$. Then $\mathbf{x}_{0}^{*} \in T_{1}^{\circ}$ and $\mathbf{A}^{N} \mathbf{x}_{0}^{*}=\mathbf{x}_{0}^{*}+\mathbf{d}$ for $\mathbf{d}=\sum_{j=0}^{N-1} \mathbf{A}^{j} \mathbf{d}_{N-j}^{*}$. Observe that $\mathbf{d} \in \mathcal{D}_{11}^{N}$. So we have

$$
-\mathbf{x}_{0}^{*} \in \mathrm{A}^{N}\left(-\mathbf{x}_{0}^{*}\right)+\mathcal{D}_{11}^{N} .
$$

Now consider the inflation functional equation $\mathcal{X}=\psi^{N}(\mathcal{X})$. Set $\mathcal{X}^{(0)}=\left(X_{1}^{(0)}, \ldots\right.$, $\left.X_{n}^{(0)}\right)$, with

$$
X_{1}^{(0)}=\left\{-\mathbf{x}_{0}^{*}\right\}, X_{2}^{(0)}=\emptyset, \ldots, X_{n}^{(0)}=\emptyset .
$$


Define $\mathcal{X}^{(m)}=\psi^{N}\left(\mathcal{X}^{(m-1)}\right)=\psi^{m N}\left(\mathcal{X}^{(0)}\right)$. Set

$$
X_{1}^{(1)}=\bigvee_{j=1}^{n}\left(\mathrm{~A}^{N}\left(X_{j}^{(0)}\right)+\mathcal{D}_{1 j}^{N}\right)=\mathrm{A}^{N}\left(\left\{\mathbf{x}_{0}^{*}\right\}\right)+\mathcal{D}_{11}^{N},
$$

hence we have $X_{1}^{(0)} \subseteq X_{1}^{(1)}$ by (6.2), and obviously we have $\emptyset=X_{i}^{(0)} \subseteq X_{i}^{(1)}$ for $i \geq 2$. So the inclusion property $\mathcal{X}^{(0)} \subseteq \psi^{N}\left(\mathcal{X}^{(0)}\right)=\mathcal{X}^{(1)}$ holds. It follows that $\mathcal{X}^{(0)} \subseteq \mathcal{X}^{(1)} \subseteq \mathcal{X}^{(2)} \subseteq \cdots$. However, notice that

$$
X_{i}^{(m)}=\bigvee_{j=1}^{n}\left(\mathrm{~A}^{m N}\left(X_{j}^{(0)}\right)+\mathcal{D}_{i j}^{m N}\right)=\mathrm{A}^{m N}\left\{\mathbf{x}_{0}^{*}\right\}+\mathcal{D}_{i 1}^{m N} .
$$

We conclude that each $X_{i}^{(m)}$ is an ordinary set and is $\varepsilon_{0}$-uniformly discrete. Let $X_{i}=$ $\bigcup_{m=0}^{\infty} X_{i}^{(m)}$ and $\hat{\mathcal{X}}=\left(X_{1}, \ldots, X_{n}\right)$. Then $\hat{\mathcal{X}}=\psi^{N}(\hat{\mathcal{X}})$, and $\hat{\mathcal{X}}$ is $\varepsilon_{0}$-uniformly discrete.

It remains to show that $\hat{\mathcal{X}}$ is a substitution Delone set family and to establish the tiling property of $\hat{\mathcal{X}}$. Observe that $\mathbf{0}$ is in the interior of $T_{1}-\mathbf{x}_{0}^{*}$. Now

$$
\mathrm{A}^{m N}\left(T_{1}-\mathbf{x}_{0}^{*}\right)=\bigcup_{j=1}^{n}\left(T_{j}+\underline{\mathcal{D}}_{j 1}^{m N}-\mathrm{A}^{m N} \mathbf{x}_{0}^{*}\right)=\bigcup_{j=1}^{n}\left(T_{j}+X_{j}^{(m)}\right) .
$$

Note that the union $\bigcup_{j=1}^{n}\left(T_{j}+\underline{\mathcal{D}}_{j 1}^{m N}\right)$ is measurewise disjoint as proved in the proof of (v) $\Rightarrow$ (i) in Theorem 5.5. Taking the limit as $m \rightarrow \infty$ will keep the union measurewise disjoint. Hence $\bigcup_{j=1}^{n}\left(T_{j}+X_{j}\right)$ is a tiling of $\mathbb{R}^{d}$. Now all $X_{j}$ must be relatively dense as a result of the primitivity of $S$, so they are Delone sets. This completes the proof.

We conclude this section by deducing Theorem 2.4 from Theorem 6.1.

Proof of Theorem 2.4. (iii) $\Rightarrow$ (ii) This follows from Theorem 6.1(iv) $\Rightarrow$ (iii).

(ii) $\Rightarrow$ (i) A self-replicating multi-tiling family is a weak substitution Delone multiset family.

(i) $\Rightarrow$ (iii) By Theorem 2.3 the inflation functional equation satisfies the Perron eigenvalue condition. A weak substitution Delone multiset family is a weakly uniformly discrete multiset family, and satisfies the Perron eigenvalue condition by Theorem 2.3. Thus the conditions of Theorem 6.1(i) hold, and the result then follows from Theorem 6.1(iv).

\section{Self-Replicating Multi-Tiling Families}

In this section we study self-replicating multi-tiling families as a subclass of weak substitution Delone multiset families.

Theorem 7.1. Let $\mathcal{X}$ be an irreducible weak Delone multiset family satisfying the inflation functional equation $\psi(\mathcal{X})=\mathcal{X}$ for the data $\left(\mathrm{A}, \mathcal{D}_{i j}\right)$, whose subdivision matrix $\mathrm{S}$ is primitive. Suppose that the fundamental cycle of $\mathcal{X}$ has period 1 . Then $\mathcal{X}$ is a self-replicating multi-tiling family. 
Proof. Since the fundamental cycle of $\mathcal{X}=\left(X_{1}, \ldots, X_{n}\right)$ has period 1, it contains a single element $\left\{\mathbf{x}_{0}\right\}$, and since it is irreducible, by Theorem 2.2 it has multiplicity one. Without loss of generality we assume that $\mathbf{x}_{0} \in X_{1}$, so $\mathbf{x}_{0}=A \mathbf{x}_{0}+\mathbf{d}$ for some $\mathbf{d} \in \mathcal{D}_{11}$. Let $\mathcal{S}^{(0)}=\left(S_{1}^{(0)}, \ldots, S_{n}^{(0)}\right)$ such that $S_{1}^{(0)}=\left\{\mathbf{x}_{0}\right\}$ and all other $S_{i}^{(0)}=\emptyset$. Define $\mathcal{S}^{(m)}:=\psi\left(\mathcal{S}^{(0)}\right)=\left(S_{1}^{(m)}, \ldots, S_{n}^{(m)}\right)$. It follows from the expression for $\psi^{m}$ given in (6.1) that

$$
S_{i}^{(m)}=\bigvee_{j=1}^{n}\left(\mathrm{~A}^{m}\left(S_{j}^{(0)}\right)+\mathcal{D}_{i j}^{m}\right)=\mathrm{A}^{m} \mathbf{x}_{0}+\mathcal{D}_{i 1}^{m} .
$$

Suppose that $\left(T_{1}, \ldots, T_{n}\right)$ is the set of self-affine multi-tiles corresponding to $\left(\mathrm{A}, \mathcal{D}_{i j}\right)$. Now by hypothesis Theorem 2.4(i) holds, so by Theorem 2.4(iii) each $T_{i}$ has positive Lebesgue measure, and each $T_{i}$ satisfies $\overline{T_{i}^{\circ}}=T_{i}$. We have

$$
\bigcup_{j=1}^{n}\left(T_{j}+S_{j}^{(m)}\right)=\bigcup_{j=1}^{n}\left(T_{j}+\mathrm{A}^{m} \mathbf{x}_{0}+\mathcal{D}_{j 1}^{m}\right)
$$

It follows from $\mathrm{A}^{m}\left(T_{1}\right)=\bigcup_{j=1}^{n}\left(T_{j}+\mathcal{D}_{j 1}^{m}\right)$ that

$$
\mathrm{A}^{m}\left(T_{1}+\mathbf{x}_{0}\right)=\bigcup_{j=1}^{n}\left(T_{j}+S_{j}^{(m)}\right) .
$$

The unions on the right side of (7.3) are all measurewise disjoint. Taking the limit $m \rightarrow \infty$ we see that $\Omega:=\bigcup_{j=1}^{n}\left(T_{j}+X_{j}\right)$ is a packing of $\mathbb{R}^{d}$.

It remains to prove that $\Omega$ is a tiling. The set $\Omega=\bigcup_{j=1}^{n}\left(T_{j}+X_{j}\right)$ is closed and satisfies

$$
\begin{aligned}
\mathrm{A}(\Omega) & =\bigcup_{j=1}^{n}\left(\mathrm{~A}\left(T_{j}\right)+\mathrm{A}\left(X_{j}\right)\right) \\
& =\bigcup_{j=1}^{n}\left(\bigcup_{i=1}^{n}\left(T_{i}+\mathcal{D}_{i j}+\mathrm{A}\left(X_{j}\right)\right)\right) \\
& =\bigcup_{i=1}^{n}\left(T_{i}+\bigcup_{j=1}^{n}\left(\mathcal{D}_{i j}+\mathrm{A}\left(X_{j}\right)\right)\right) \\
& =\bigcup_{i=1}^{n}\left(T_{i}+X_{i}\right)=\Omega .
\end{aligned}
$$

We now argue by contradiction, and suppose $\Omega \neq \mathbb{R}^{d}$. Since $\Omega$ is closed, there exists a ball $B$ of radius $\varepsilon>0$ such that $B \cap \Omega=\emptyset$, which yields $\mathrm{A}^{N}(B) \cap \mathrm{A}^{N}(\Omega)=\mathrm{A}^{N}(B) \cap \Omega=\emptyset$. However, $\mathrm{A}$ is expanding, so by taking $N$ sufficiently large $\mathrm{A}^{N}(B)$ contains a ball of arbitrarily large radius. This ball is disjoint from $\Omega$, so it is not filled by any translate $T_{j}+\mathbf{x}_{j}, \mathbf{x}_{j} \in X_{j}$ and $1 \leq j \leq n$. Therefore $X_{j}$ cannot be a weak Delone set, a contradiction. Thus we have a tiling. 
Remarks. (1) There exist irreducible self-replicating multi-tiling families whose fundamental cycles have period exceeeding 1, see Example 8.7. Thus the condition of Theorem 7.1 is only a sufficient condition to give a self-replicating multi-tiling family.

(2) Let $\mathcal{X}$ be an irreducible Delone set family which satisfies an inflation functional equation $\psi(\mathcal{X})=\mathcal{X}$ with a primitive subdivision matrix $S$ and has a fundamental cycle of period $p$. By Theorems 2.3 and 6.1 the unique solution $\mathcal{T}:=\left(T_{1}, \ldots, T_{n}\right)$ consisting of compact sets of the associated multi-tile equation has $T_{i}$ of positive measure, which are the closure of their interiors. The set $\mathcal{T}+\mathcal{X}:=\bigcup_{i=1}^{n}\left(T_{i}+X_{i}\right)$ gives a partial $q$ packing of $\mathbb{R}^{d}$ for some $q \leq p$, using the tiles $T_{i}$. That is, each point of $\mathbb{R}^{d}$ is covered with multiplicity at most $q$ off a set of measure zero and a set of infinite measure has multiplicity exactly $q$, with possibly another set of infinite measure of strictly smaller multiplicity.

One expects that in many cases this construction produces a $p$-thick multi-tiling of $\mathbb{R}^{d}$. We formulate the following problem:

Problem. Let $\mathcal{X}$ be an irreducible Delone set family which satisfies an inflation functional equation $\psi(\mathcal{X})=\mathcal{X}$ with a primitive subdivision matrix S. Suppose $\mathcal{X}$ is uniformly discrete and has a fundamental cycle of period $p$. Is it true that $\mathcal{T}+\mathcal{X}:=$ $\bigcup_{i=1}^{n}\left(T_{i}+X_{i}\right)$ is always a $q$-thick multi-tiling for some $q \leq p$ ? If not, what extra conditions are needed to ensure it?

\section{Examples}

Example 8.1 (Substitution Multiset with Unbounded Multiplicity Function). Let $\mathrm{A}=$ [3] and $\mathcal{D}_{1,1}=\{0,1,2,3\}$, so that $\left|\mathcal{D}_{1,1}\right|=4>3=|\operatorname{det}(\mathrm{A})|$. Take the seed $\mathcal{S}^{(0)}=$ $S_{1}^{(0)}=\{0\}$. Then the inclusion property $\mathcal{S}^{(0)} \subseteq \mathcal{S}^{(1)}$ holds, hence

$$
X=X_{1}=\lim _{k \rightarrow \infty} S_{1}^{(k)}
$$

defines a multiset $X$. The multiset $X \subseteq \mathbb{Z}_{\geq 0}$ and each point $l \in \mathbb{Z}^{+}$occurs with finite multiplicity $m_{X}(l)$. One can verify that $m_{X}\left(3^{k}\right)=k+1$, which shows that $m(l)$ is unbounded as $l \rightarrow \infty$. This example corresponds to case (ii) in Theorem 3.5.

Example 8.2 (Discrete Substitution Set Family that Is Not Uniformly Discrete). Let $\mathrm{A}=[3]$ and $\mathcal{D}_{1,1}=\{0,1, \pi\}$, with $\pi=3.14159 \ldots$ Take the seed $\mathcal{S}^{(0)}=S_{1}^{(0)}=\{0\}$. Then $\mathcal{S}^{(0)} \subseteq \mathcal{S}^{(1)}$ and the limit

$$
X_{1}=\lim _{k \rightarrow \infty} S_{1}^{(k)}
$$

exists. In this case the multiset $X_{1} \subseteq \mathbb{R}_{\geq 0}$ is discrete, and its elements all have multiplicity one. It is easy to show that it has linear growth. Indeed, the $2 \cdot 3^{n-1}$ elements in $S_{1}^{(n)} \backslash S_{1}^{(n-1)}$ all satisfy

$$
3^{n} \leq x \leq \pi\left(3^{n}+3^{n-1}+\cdots+3+1\right) \leq 2 \pi \cdot 3^{n} .
$$


The associated multi-tile functional equation is

$$
\mathrm{A}\left(T_{1}\right)=T_{1} \cup\left(T_{1}+1\right) \cup\left(T_{1}+\pi\right) .
$$

The compact solution $T_{1}$ to this equation has Lebesgue measure zero, see [12] or [17]. It follows from Theorem 6.1 that $X_{1}$ cannot be uniformly discrete.

Example 8.3 (Inflation Functional Equation Having Infinitely Many Discrete Solutions). Consider the inflation functional equation on $\mathbb{R}$ with $\mathrm{A}=[2], n=1$ and $\mathcal{D}_{1,1}=\{0,1\}$. This satisfies the hypotheses of Lemma 3.7. The associated tile is $T_{1}=[0,1]$. The allowed starting points for a cycle $Y$ of period $p$ (an arbitrary positive integer) are given by (3.16), which gives

$$
x_{0}=-\frac{m}{2^{p}-1} \quad \text { for } \quad 0 \leq m \leq 2^{p}-1
$$

Each such $x_{0}$ generates an irreducible multiset $\mathcal{X}_{m, p}$ consisting of a single multiset $X_{1}=X_{1}(m, p)$ which has all multiplicities one. Now suppose that $p \geq 2$ and restrict to those $x_{0}=-m /\left(2^{p}-1\right)$ in a cycle of minimal period $p$, with $p \geq 2$. This always happens when $\operatorname{gcd}\left(m, 2^{p}-1\right)=1$, and possibly in other cases as well, but rules out the cases $m=0$ and $m=2^{p}-1$, which generate minimal cycles of period 1 , having the symbolic dynamics $d_{1} d_{2} \cdots d_{p}=0^{p}$ and $1^{p}$, respectively. Thus $-1<x_{0}<0$, so that the tile $T_{1}+x_{0}$ includes 0 in its interior, and the same holds for the other $p-1$ tiles in the periodic cycle. We conclude that the cycles of minimal period $p$ generate a multiple tiling of $\mathbb{R}$ with thickness $p$, using copies of the tile $T_{1}$.

Example 8.4 (Inflation Functional Equation Having No Nonempty Discrete Multiset Solution). Consider the inflation functional equation on $\mathbb{R}$ with $\mathrm{A}=[2], n=2$ with digit sets $\mathcal{D}_{11}=\mathcal{D}_{12}=\mathcal{D}_{21}=\mathcal{D}_{22}=\{0,1\}$. This data satisfies the hypotheses of Lemma 3.7, but the inflation functional equation $\psi(\mathcal{X})=\mathcal{X}$ has no discrete multiset solution. If fact, all elements in $\mathcal{X}$ must have infinite multiplicity. To see this we iterate the inflation functional equation $m$ times to obtain

$$
X_{i}=\left(X_{1}+\mathcal{D}_{i 1}^{m}\right) \vee\left(X_{2}+\mathcal{D}_{i 2}^{m}\right), \quad i=1,2,
$$

where each multiset $\mathcal{D}_{i j}^{m}$ has an underlying set $\underline{\mathcal{D}}_{i j}^{m}=\left\{0,1, \ldots, 2^{m}-1\right\}$ with each element having multiplicity $2^{m}$. Therefore the multiplicity of each element in $X_{i}$ is at least $2^{m}$. Since $m$ is unbounded, no element in any $X_{i}$ can have a finite multiplicity.

Example 8.5 (Irreducible Weak Substitution Delone Multiset Family with Bounded Multiplicities, Some Multiplicities Exceeding One). Consider the inflation functional equation $A=[3]$ on $\mathbb{R}$, with $n=2$ and digit sets $\mathcal{D}_{1,1}=\{\pi+3\}, \mathcal{D}_{1,2}=\{1\}$, $\mathcal{D}_{2,1}=\{-3, \pi\}, \mathcal{D}_{2,2}=\emptyset$. The subdivision matrix

$$
S=\left[\begin{array}{ll}
1 & 1 \\
2 & 0
\end{array}\right]
$$

is primitive, and its Perron eigenvalue $\lambda(S)=2$. We claim that the cycle $Y=\{0 \in$ $X_{1}, 1 \in X_{2}$ \} of period $p=2$, both points of multiplicity one, generates a discrete 
irreducible multiset family $\mathcal{X}=\left(X_{1}, X_{2}\right)$. The only point reached in one step by exiting from the cycle is $y=\pi+3 \in X_{1}$, which is reached in two different ways, one from $0 \in X_{1}$ and one from $1 \in X_{2}$, so has multiplicity two. All other points are descendants of $y$, and necessarily fall in the interval $[\pi+3, \infty)$. All the maps $x \rightarrow 3 x+d$ for $d \in \mathcal{D}_{i, j}$ are expanding outside the interval $[-4,4]$ with an expansion factor at least 1.4 , so it follows that $\mathcal{X}=\left(X_{1}, X_{2}\right)$ exists, and is irreducible and discrete. We claim that the descendants of $y$ are distinct and consequently all have multiplicity two. To show they are distinct, note that all descendants of $y$ have the form $x=\left(3^{k} a_{0}+3^{k-1} a_{1}+\cdots+3 a_{k-1}+a_{k}\right) \pi+r$, in which $a_{0}=1$ and each subsequent $a_{i}=0$ or 1 , and $r$ is an integer. The sequence $\left(a_{0}, a_{1}, \ldots, a_{k}\right)$ completely specifies the digit sequence leading to $x$, which determines which of $X_{1}$ and $X_{2}$ the point $x$ belongs to; furthermore, every such digit sequence is legal. Since all integers $3^{k} a_{0}+3^{k-1} a_{1}+\cdots+3 a_{k-1}+a_{k}$ are distinct, and $\pi$ is irrational, we conclude that all $x$ are distinct. Thus all points in $X_{1}$ and $X_{2}$ have multiplicity two, except the generating cycle $Y$, whose two points have multiplicity one.

Example 8.6 (Substitution Delone Set that Is Not Self-Replicating). Let $A=[3]$ and $\mathcal{D}_{1,1}=\{-1,0,1\}$. The associated tile $T_{1}=\left[-\frac{1}{2}, \frac{1}{2}\right]$, which consists of all balanced ternary expansions

$$
x=\sum_{j=1}^{\infty} \mathbf{d}_{j} 3^{-j}, \quad \mathbf{d}_{j} \in\{-1,0,1\}
$$

The set $\mathcal{S}^{(0)}=\left\{x_{0}=-\frac{1}{8}, x_{1}=-\frac{3}{8}\right\}$ has

$$
\mathcal{S}^{(0)} \subseteq \psi\left(\mathcal{S}^{(0)}\right)=\left\{-\frac{17}{8},-\frac{11}{8},-\frac{9}{8},-\frac{3}{8},-\frac{1}{8}, \frac{5}{8}\right\},
$$

hence $\mathcal{S}^{(0)}$ generates an irreducible discrete multiset family $\mathcal{X}=\left\{X_{1}\right\}$ which consists of the single set $X_{1}$ given by

$$
X_{1}=\sum_{k \rightarrow \infty} \mathcal{S}^{(k)}
$$

The set $X_{1}$ is irreducible and $\mathcal{S}_{0}$ is its generating cycle of period 2. A calculation gives

$$
X_{1}=\left(-\frac{3}{8}+\mathbb{Z}\right) \cup\left(-\frac{1}{8} \cup \mathbb{Z}\right) .
$$

It is a Delone set, and $X_{1}+T$ is a multiple tiling of $\mathbb{R}$ of thickness 2 . The thickness equals the period of the generating cycle, since both elements of $\mathcal{S}^{(0)}$ lie in the interior of the tile $T_{1}$. Thus $X_{1}$ is a substitution Delone set but not a self-replicating Delone set.

Example 8.7 (Self-Replicating Delone Set Family Having a Primitive Cycle of Order Larger Than One). Let $\mathrm{A}=[-2]$ and $\mathcal{D}_{11}=\{-2,-1\}$. Then $\mathcal{X}=\left(X_{1}\right)$ with $X_{1}=\mathbb{Z}$ is an irreducible substitution Delone set family whose fundamental cycle is $\{0,-1\}$ and has period 2. The corresponding self-affine tile is $T_{1}=[0,1]$. So $\mathcal{T}+\mathcal{X}=T_{1}+X_{1}$ tiles $\mathbb{R}$, hence is a self-replicating Delone set.

Example 8.8 (Nonperiodic and Aperiodic Self-Replicating Delone Set Families). A Delone set $X$ is (fully) periodic if it has a full rank lattice of periods $\Lambda_{X}=\{\mathbf{t}: X=X+\mathbf{t}\}$. 
It is nonperiodic if it is not fully periodic and it is aperiodic if it has no periods, i.e., $\Lambda_{X}=\{\mathbf{0}\}$. An example of a two-dimensional self-replicating Delone set, which is not fully periodic, but has a one-dimensional lattice of periods, was given in Example 2.3 of [18]. Recently Lee and Moody [21] constructed many self-replicating Delone sets which are nonperiodic, including aperiodic examples, whose points are contained in a lattice in $\mathbb{R}^{d}$. They give such examples associated to nonperiodic tilings including the sphinx tiling of Godreche [3] and the chair tiling.

\section{Acknowledgments}

We are indebted to the reviewers for very detailed comments improving the paper.

\section{References}

1. T. Flaherty and Y. Wang, Haar-type multiwavelet bases and self-affine multi-tiles, Asian J. Math. 3(2) (1999), 387-400.

2. F. Gähler and R. Klitzing, The diffraction pattern of self-similar tilings, in: Mathematics of Long-Range Aperiodic Order (R. V. Moody and J. Patera, Eds.), Kluwer: Norwich, MA, 1997, pp. 141-174.

3. C. Godreche, The sphinx: a limit periodic tiling of the plane, J. Phys. A 22 (1989), L1163-L1168.

4. K. Gröchenig, A. Haas, and A. Raugi, Self-affine tilings with several tiles, I, Appl. Comput. Harmon. Anal. 7 (1999), 211-238.

5. K. Gröchenig and W. Madych, Multiresolution analysis, Haar bases and self-similar tilings, IEEE Trans. Inform. Theory IT-38(2) (1992), 556-568.

6. A. Hof, Quasicrystals, Aperiodicity and Lattice Systems, Thesis, University of Groningen, 1992.

7. C. Janot, Quasicrystals: A Primer, Oxford University Press: Oxford, 1992.

8. R. Kenyon, Self-Similar Tilings, Ph.D. thesis, Princeton University, Princeton, NJ, 1990.

9. R. Kenyon, Self-replicating tilings, in: Symbolic Dynamics and Its Applications (P. Walters, Ed.), Contemp. Math. Vol. 135, AMS: Providence, RI, 1992, pp. 239-264.

10. R. Kenyon, Inflationary tilings with a similarity structure, Comment. Math. Helv. 69 (1994), 169-198.

11. R. Kenyon, The construction of self-similar tilings, GAFA 6 (1996), 471-488.

12. R. Kenyon, Projecting the one-dimensional Sierpinski gasket, Israel J. Math. 97 (1997), 221-238.

13. D. Knuth, The Art of Computer Programming, Vol. II. Seminumerical Algorithms, Second Edition, Addison-Wesley: Reading, MA, 1981.

14. J. C. Lagarias, Meyer's concept of quasicrystal and quasiregular sets, Comm. Math. Phys. 179 (1996), 365-376.

15. J. C. Lagarias, Geometric models for quasicrystals, I. Delone sets of finite type, Discrete Comput. Geom. 21 (1999), 161-191.

16. J. C. Lagarias, Geometric models for quasicrystals, II. Local rules under isometries, Discrete Comput. Geom. 21 (1999), 345-372.

17. J. C. Lagarias and Y. Wang, Tiling the line with translates of one tile, Invent. Math. 124 (1996), 341-365.

18. J. C. Lagarias and Y. Wang, Self-affine tiles in $\mathbb{R}^{n}$, Adv. in Math. 121 (1996), 21-49.

19. J. C. Lagarias and Y. Wang, Integral self-affine tiles in $\mathbb{R}^{n}$, I. Standard and nonstandard digit sets, J. London Math. Soc. 54 (1996), 161-179.

20. J. C. Lagarias and Y. Wang, Integral self-affine tiles in $\mathbb{R}^{n}$, II. Lattice tilings, J. Fourier Anal. Appl. 3 (1997), 83-102.

21. J.-Y. Lee and R. V. Moody, Lattice substitution systems and model sets, Discrete Comput. Geom. 25 (2001), 173-202.

22. D. Levine and P. J. Steinhardt, Quasicrystals: a new class of ordered structures, Phys. Rev. Lett. 53 (1984), 2477-2480. 
23. D. Lind, Dynamic properties of quasihyperbolic toral automorphisms, Ergodic Theory Dynamical Systems 2 (1982), 49-68.

24. W. F. Lunnon and P. A. B. Pleasants, Quasicrystallographic tilings, J. Math. Pures Appl. 66 (1987), 217-263.

25. R. D. Mauldin and S. Williams, Hausdorff dimension in graph directed constructions, Trans. Amer. Math. Soc. 309 (1988), 811-829.

26. Y. Meyer, Quasicrystals, diophantine approximation and algebraic numbers, in: Beyond Quasicrystals (F. Axel and D. Gratias, Eds.), Les Editions de Physique, Springer-Verlag: New York, 1995, pp. 3-16.

27. R. V. Moody, Meyer sets and the finite generation of quasicrystals, in: Symmetries in Science VIII (B. Gruber, Ed.), Plenum: New York, 1995.

28. R. V. Moody, Meyer sets and their duals, in: The Mathematics of Long Range Aperiodic Order (R. V. Moody and J. Patera, Eds.), Kluwer: Norwell, MA, 1997, pp. 403-441.

29. B. Praggastis, Markov partitions for hyperbolic toral automorphisms, Ph.D. Thesis, University of Washington, 1992.

30. B. Praggastis, Numeration systems and Markov partitions from self-similar tilings, Trans. Amer. Math. Soc. 351 (1999), 3315-3349.

31. M. Queffélec, Substitution Dynamical Systems - Spectral Analysis, Lecture Notes in Mathematics No. 1294, Springer-Verlag: New York, 1987.

32. E. A. Robinson, Jr., The dynamical theory of tilings and quasicrystallography, in: Ergodic Theory of $\mathbb{Z}^{n}$-Actions (M. Pollicott and K. Schmidt, Eds.), London. Math. Soc. Lecture Notes No. 228, Cambridge University Press: Cambridge, 1996, pp. 451-473.

33. M. Senechal, Quasicrystals and Geometry, Cambridge University Press: Cambridge, 1995.

34. V. Sirvent and Y. Wang, Self-affine tilings via substitution dynamical systems and Rauzy fractals, Pacific J. Math. 206 (2002), 465-486.

35. B. Solomyak, Dynamics of self-similar tilings, Ergodic Theory Dynamical Systems 17 (1997), 695-738. (Corrections: ibid. 19 (1999), 1685.)

36. B. Solomyak, Non-periodicity implies unique composition for self-similar translationally finite tilings, Discrete Comput. Geom. 20 (1998), 265-279.

37. B. Solomyak, Spectrum of dynamical systems arising from Delone sets, in: Quasicrystals and Discrete Geometry (J. Patera, Ed.), American Mathematical Society: Providence, RI, 1998, pp. 265-275.

38. C. Stein, Singular Integrals and Differentiability Properties of Functions, Princeton University Press: Princeton, NJ, 1970.

Received September 4, 2001, and in revised form May 15, 2002. Online publication December 20, 2002. 